\title{
Data-Driven Model-Free Adaptive Control of Particle Quality in Drug Development Phase of Spray Fluidized-Bed Granulation Process
}

\author{
Zhengsong Wang, ${ }^{1}$ Dakuo He, ${ }^{1,2}$ Xu Zhu, ${ }^{1}$ Jiahuan Luo, ${ }^{1}$ Yu Liang, ${ }^{1}$ and Xu Wang ${ }^{3}$ \\ ${ }^{1}$ College of Information Science and Engineering, Northeastern University, Shenyang, Liaoning 110004, China \\ ${ }^{2}$ State Key Laboratory of Synthetical Automation for Process Industries, Northeastern University, Shenyang 110004, China \\ ${ }^{3}$ State Key Laboratory of Process Automation in Mining \& Metallurgy, Beijing 100160, China \\ Correspondence should be addressed to Dakuo He; hedakuo@ise.neu.edu.cn
}

Received 28 September 2017; Accepted 19 November 2017; Published 12 December 2017

Academic Editor: Julio Blanco-Fernández

Copyright ( 2017 Zhengsong Wang et al. This is an open access article distributed under the Creative Commons Attribution License, which permits unrestricted use, distribution, and reproduction in any medium, provided the original work is properly cited.

A novel data-driven model-free adaptive control (DDMFAC) approach is first proposed by combining the advantages of modelfree adaptive control (MFAC) and data-driven optimal iterative learning control (DDOILC), and then its stability and convergence analysis is given to prove algorithm stability and asymptotical convergence of tracking error. Besides, the parameters of presented approach are adaptively adjusted with fuzzy logic to determine the occupied proportions of MFAC and DDOILC according to their different control performances in different control stages. Lastly, the proposed fuzzy DDMFAC (FDDMFAC) approach is applied to the control of particle quality in drug development phase of spray fluidized-bed granulation process (SFBGP), and its control effect is compared with MFAC and DDOILC and their fuzzy forms, in which the parameters of MFAC and DDOILC are adaptively adjusted with fuzzy logic. The effectiveness of the presented FDDMFAC approach is verified by a series of simulations.

\section{Introduction}

Spray fluidized-bed granulation process (SFBGP) is a process that forms small particles into larger granules using the liquid binding solution sprayed onto fluidized particles by a spray nozzle above the powder bed [1]. Because of advantage of single unit operation, SFBGP has been widely applied to produce granules aiming at improving power flowability and physicochemical properties of drugs $[2,3]$. As a key technique in the production of pharmaceutical solid dosage forms of tablets and capsules, the primary target of SFBGP is to produce granules with consistent product quality for the following pharmaceutical processes. Therefore, particle quality control of SFBGP is of great theoretical and practical significance.

For a SFBGP, the quality of particles can be evaluated by many factors such as production yield, drug content, size, density, friability, flowability, and compressibility $[2,4]$, and among them granule size is the key characteristic to control [5-8]. To achieve the desired granule size, model-based control (MBC) schemes, which require a priori physical and mathematical knowledge of the process, may be the effective techniques. Previous works have introduced MBC frameworks and examined the implementation of $\mathrm{MBC}$ strategies on conventional granulation design [3, 9-13].

It is well known that SFBGP is a complicated process that is significantly influenced by both the material-related factors such as the nature and characteristics of powder particles and binding agents and the process factors associated with granulation such as fluidizing air velocity and binder feed rate $[2,3,5,7,14,15]$. In the drug development phase, however, the frequent adjustment of prescription will give rise to the variation of material attributes. So for a brand-new prescription, the operating condition space should be redesigned in order to accurately and rapidly achieve the desired particle quality. But for such a SFBGP whose material attributes have already changed and that we have never encountered before, the absence of process operating experience and historical data leads to great difficulty in developing accurate 
process models. If traditional $\mathrm{MBC}$ schemes are used, lots of experiments in the actual process should first be implemented as long as the prescription is changed to get enough process data used for developing precise mathematical models. Such a way is laborious, time-consuming, and resource-wasting, so that the $\mathrm{MBC}$ techniques are no more applicable to quality control task in this work.

Data-driven control (DDC) $[16,17]$ means that only the input/output (I/O) measurement data of controlled plant are used in controller design. DDC approaches do not require a model of a plant, and the modeling process, the unmodeled dynamics, and the theoretical assumptions all disappear [1619]. Therefore, DDC has attracted considerable attention in recent years [18], and there are many DDC approaches together with their practical applications in many fields that could be found in the literature, like the following: modelfree adaptive control (MFAC) [16, 17, 20-24], data-driven optimal iterative learning control (DDOILC) [25-27], virtual reference feedback tuning [28-30], lazy learning control [31], dynamic programming methods [32], and others [33-36]. In spite of this, to the best of authors' knowledge, there is no report about the research on application of DDC to SFBGP that has been published in the literature. In this work, a study on particle quality control based on DDC approaches in the drug development phase of a SFBGP is conducted to resolve the practical difficulty encountered in redesigning operating condition when prescription and material attributes are all changed.

The mechanism model of SFBGP should first be introduced to conduct such quality control research. Such a process model plays several significant roles: first, modeling analyzes the mechanism of SFBGP, identifies the important manipulated variables and quality indices, and establishes the relationship between them. Second, such a model can be used as a simulator of a real SFBGP to generate the required process data used for simulation and analysis. In this work, a widely accepted mechanistic model for SFBGP $[37,38]$ is introduced to simulate the actual SFBGP and produce the required process data.

In this work, we select two classical and representative DDC approaches, MFAC and DDOILC, to study data-driven model-free adaptive control (DDMFAC) of average particle size (APS) for SFBGP with simulation experiment research. MFAC algorithm is proposed by Hou and Jin [16, 17] based on a new dynamic linearization technique (DLT) and then applied in several areas [18]. The main feature of MFAC is that the controller design depends merely on the $\mathrm{I} / \mathrm{O}$ measurement data of the controlled plant. Instead of identifying a nonlinear process model of a plant, an equivalent local dynamical linearization model is constructed along the dynamic operation points of the system using the DLT with a novel concept called pseudo-partial derivative (PPD). The time-varying PPD could be estimated merely using the I/O measurement data of the controlled plant. DDOILC is developed and applied to both linear and nonlinear systems by Chi et al. [25]. For this approach, the only required knowledge of a controlled system is that the Markov matrices of linear systems or the partial derivatives of nonlinear systems with respect to control inputs are bounded. DDOILC is actually the extension of MFAC, and these two approaches have the same systematic framework. Compared with other DDC methods, MFAC and DDOILC have several attractive advantages that make them more suitable for many practical control applications [16]. First, they do not require any process model and structural information of the controlled plant and merely depend on the real time measurement $\mathrm{I} / \mathrm{O}$ data of the controlled plant, which indicates that it is feasible to independently design a generic controller for a certain class of practical industrial processes. Second, they are lower cost controllers because they do not need any training process. Third, they are simple and easily implemented with small computational burden and have strong robustness. Finally, they have been successfully implemented in many practical fields, such as chemical industry [20,21, 25, 26], linear motor control and injection moulding process [22], $\mathrm{PH}$ value control [23], and robotic welding process [24].

The controllers of MFAC and DDOILC all consist of a control input iterative learning law and a PPD matrix iterative updating law. From this respect, the prime difference between them is whether or not to use the predicted data of system output of current iteration in controller design. In MFAC, the predicted system output of current iteration is utilized, but merely the output measurement data of previous iterations are used in DDOILC, which makes their different characteristics. Through theory analysis, MFAC and DDOILC both have advantages with regard to different initial values (IVs) for particle quality. When IV is far away from desired particle quality, that is, the initial tracking error is larger, DDOILC has quicker convergence speed. The reason for this is that the utilization of actual output of previous iterations in controller design of DDOILC will give larger updating step size for control input and PPD vector. However, as IV gets closer to desired output, MFAC will gradually show its advantages since the prediction accuracy of its dynamic linearization model (DLM) is getting higher and higher. To verify the conclusion obtained through theoretical analysis, we conducted several comparative experimental studies by constructing a series of IVs, and the simulation results validate our conclusion.

In the drug development phase, the frequent variations of prescription will lead to diversity of material properties. For different material properties, the same group of empirical operating conditions will give entirely different IVs of particle quality. It is demanded that the DDC approaches must accommodate various IVs in the practice. Therefore, to further improve the overall control effect, a novel hybrid DDC approach, which is named DDMFAC and combines the advantages of MFAC and DDOILC, is presented in this paper. The newly designed controller is able to exhibit the features of MFAC and DDOILC in different extent by adjusting their weighted factors. Compared with MFAC and DDOILC, however, even DDMFAC does not have overwhelming superiority for all IVs. Simulation results have revealed that DDMFAC may be inferior to DDOILC or MFAC in particle quality control if there is no reasonable adjustment strategy of weighted parameters. By the previous theoretical analysis for MFAC and DDOILC and the subsequent simulation verification, we can sum up weighted parameters adjustment 


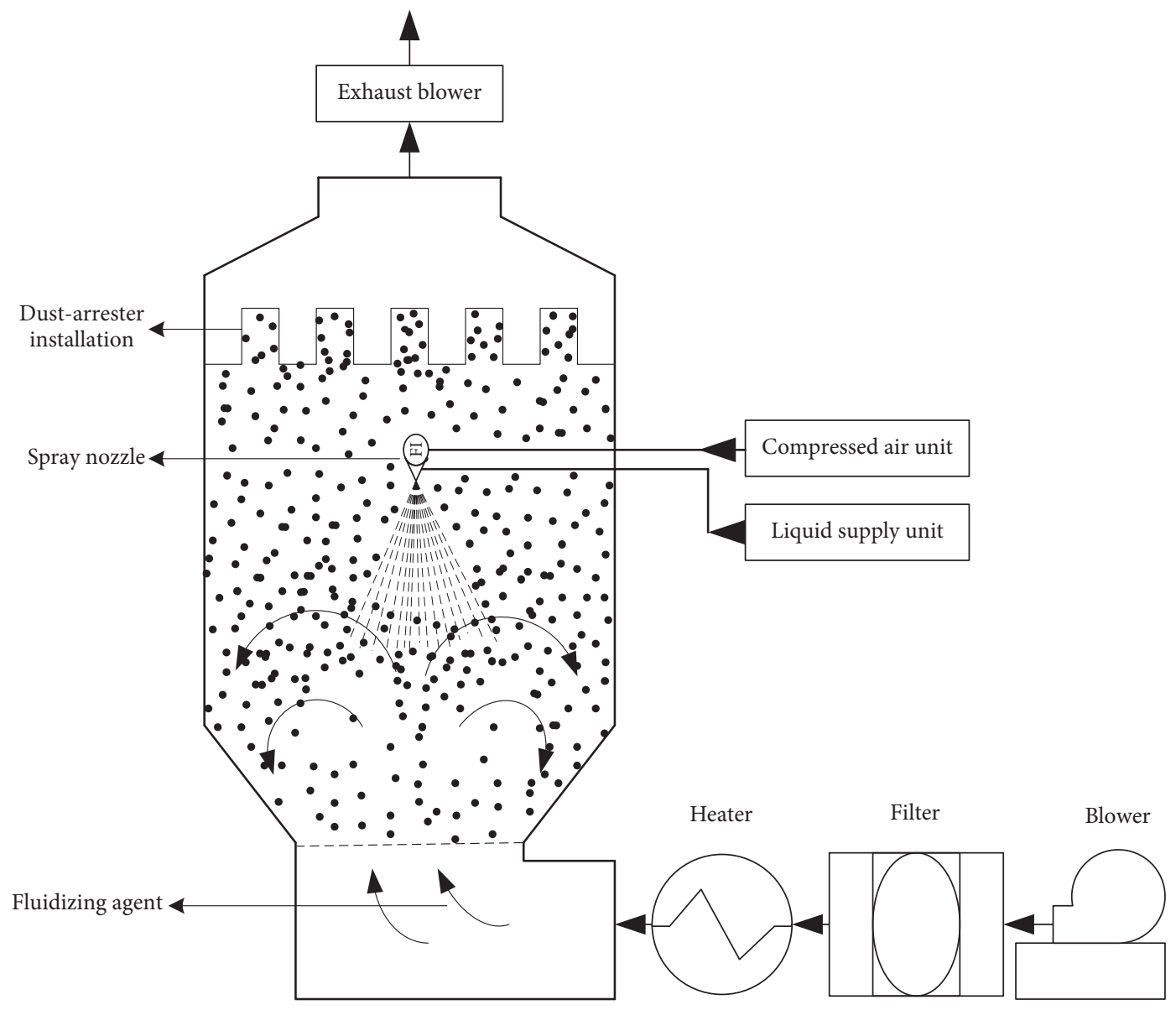

FIGURE 1: The schematic diagram of SFBGP.

rules for DDMFAC. Then fuzzy adaptive adjustment of target weighted parameters is implemented with the aim of rapidly converging DDMFAC by making full use of the advantage of MFAC or DDOILC at different control stages. We named the final DDC approach presented in this paper as fuzzy DDMFAC (FDDMFAC), and a series of simulations validate its effectiveness.

\section{Preliminaries}

2.1. Mathematical Mechanism Model of Spray Fluidized-Bed Granulation Process. SFBGP is a commonly used unit operation in the pharmaceutical industry. The powders can be mixed, granulated, and dried in the same equipment, which minimizes the equipment costs, loss of product transfers, and possibility of the cross-contamination [3]. The rationale of spray fluidized-bed granulation can be briefly described as follows: (1) Particles circulate within granulator by pumping hot air from the bottom of granulator and meanwhile binder liquid is sprayed on the fluidized particles in the forms of small droplets [38]. (2) As a result of collisions and coalescence between the surface-wetted particles, liquid bridges are formed and aggregation of particles occurs leading to the growth of granules $[1,39]$.
The schematic diagram of SFBGP is shown in Figure 1. The overall granulation process in fluidized-bed spray granulator is divided into three stages: powder mixing, granulation, and drying. Firstly, mixed raw materials for drug and ingredients are put in granulator. Passing through the filter and heater, hot air pumped in by blower is pumped into granulator from the bottom of granulator, which forces the fluidization of powder to sufficiently blend materials. Afterwards, liquid binder is pumped into spray nozzle from liquid supply unit and atomized into droplets by compressed air that is simultaneously pumped into spray nozzle from compressed air unit. The droplets are dispersed over the surface of fluidized particles, which contributes to the agglomeration of colliding and surface-wetted powder particles to form granules. Finally, the granules are dried to a predetermined moisture content level. The dust-arrester installation is used to prevent powder particles that are not in contact with droplets from being carried out by off-gas.

PBMs have been widely used for modeling SFBGP, in which the granule size density distribution evolves as a function of time. The population balance is a number balance around each size fraction of the size distribution based on number conservation law. The rate of change of the number of particles in a given size interval is equal to the rate at which 
the granules enter and leave that size interval. The mathematical mechanism model of SFBGP is developed based on following assumptions: (1) Each particle is spherical. (2) The total volume of the particles is conserved. (3) The particle size in a certain particular size interval is represented by the left boundary of the interval. (4) The relative growth rate is uniform for each particle within the same volume interval. A one-dimensional PBM that describes the rate of change in particle number density function $n(t, l)$ is given by

$$
\begin{aligned}
& \frac{\partial n(t, l)}{\partial t}=\frac{1}{2} \\
& \quad \cdot \int_{0}^{l} \frac{\beta\left(t,\left(l^{3}-\mu^{3}\right)^{1 / 3}, \mu\right) n\left(t,\left(l^{3}-\mu^{3}\right)^{1 / 3}\right) n(t, \mu)}{\left(l^{3}-\mu^{3}\right)^{2 / 3}} d \mu \\
& \quad-n(t, l) \int_{0}^{\infty} \beta(t, l, \mu) n(t, \mu) d \mu
\end{aligned}
$$

where $n(t, l)$ is the particle number density function in terms of particle diameter, $l$, and $\beta$ is the aggregation model $[37,38]$. $\beta(t, l, \mu)$ can generally be partitioned into size dependent and size independent parts $[3,37,38,40,41]$,

$$
\beta(t, l, u)=\beta_{0}(t) \beta^{*}(l, \mu),
$$

where $\beta_{0}(t)$ is the granulation rate constant that can be thought of as an aggregation efficiency and depends on all parameters involved in the aggregation process except particle size, such as the operating condition and binder properties. The latter term $\beta^{*}(l, \mu)$ reflects the influence of particle size on the likelihood of aggregation.

In order to solve a PBM in (1), the discretized approach $[42,43]$ is applied to determine the change in number of particles $N_{i}$ in interval $i$ as

$$
\begin{aligned}
\frac{\mathrm{d} N_{i}}{\mathrm{~d} t}= & \sum_{j=1}^{i-2} 2^{j-i+1} \beta_{i-1, j} N_{i-1} N_{j}+\frac{1}{2} \beta_{i-1, i-1} N_{i-1}^{2} \\
& -N_{i} \sum_{j=1}^{i-1} 2^{j-i} \beta_{i, j} N_{j}-N_{i} \sum_{j=i}^{n_{\max }} \beta_{i, j} N_{j},
\end{aligned}
$$

where $N_{i}$ is the number of particles within particle size range $\left(L_{i}, L_{i+1}\right) ; L_{i}$ and $L_{i+1}$ are the lower and upper limits of $i$ th particle size interval, $i=1,2, \ldots, n_{\max }$; and $n_{\max }$ is the total number of particle size intervals with a value of 12 in this paper. In the discretization scheme, the length domain of particles is divided into geometric intervals in the way that the upper and lower limits of each size interval are in a ratio of $r=L_{i+1} / L_{i}=\sqrt[3]{2}$. The size range used in modeling is from $50 \mu \mathrm{m}$ to $800 \mu \mathrm{m}$ which is divided into 12 intervals. The function "ode 45 " in MATLAB is used to solve (3) to obtain the final number of particles in each size interval. Then the APS of final granules $D_{m}$ is calculated by the following formula:

$$
D_{m}=\sum_{i=1}^{n_{\max }} V\left(N_{f, i}\right) d_{p i},
$$

where $V\left(N_{f, i}\right)$ is the volume fraction of end granules at size interval $i=1,2, \ldots, n_{\max } ; N_{f, i}$ is the number of end granules in the $i$ th size interval; and $d_{p i}$ is the geometric mean of lower limit and upper limit of $i$ th size interval, $i=1,2, \ldots, n_{\max }$.

We have just briefly introduced the general framework of PBM-based mechanism model of SFBGP. Detailed derivation process and setting of model parameters are given in $[37,38]$, and they will not be covered here. The developed mechanism model is capable of not only linking APS of particles with the operating variable (binder spray rate), but also reflecting the relationship between APS and material attributes, such as viscosity of binder and particle density. Hence, this mechanism model can be used for simulating the practical challenges in particle quality control of SFBGP. For example, a series of IVs can be constructed by changing the material attributes while keeping the operating condition unchanged. To veritably simulate the actual granulation process, the input control curve should be time-varying. But to simplify things, the input control curve is divided into three stages in the whole granulation process in this work.

2.2. The Data-Driven Control Approaches Used in This Work. As the classic and representative DDC methods, MFAC and DDOILC have some merits that make them more competitive in practical control applications. Therefore, in view of these merits, MFAC and DDOILC are selected and applied to perform data-driven particle quality control for SFBGP in this work. Now we first briefly introduce their controller design procedures. For details, please refer to [16, 25].

A repeatable batch process system to be controlled is given as follows:

$$
y(k)=f(\mathbf{u}(k)),
$$

where $y(k)$ and $\mathbf{u}(k)$ are the system output and input at $k$ th iteration, respectively, $k$ is the number of iterations, and $f(\bullet)$ is an unknown nonlinear function. This nonlinear system (5) satisfies the following assumptions:

(1) The derivatives of $f(\bullet)$ with respect to control input $\mathbf{u}(k)$ are continuous.

(2) System (5) is generalized Lipschitz; that is, $\mid \Delta y(k+$ $1) \mid \leq b\|\Delta \mathbf{u}(k)\|$ for any $k$ and $\|\Delta \mathbf{u}(k)\| \neq 0$, where $\Delta y(k+1)=y(k+1)-y(k), \Delta \mathbf{u}(k)=\mathbf{u}(k)-\mathbf{u}(k-1)$, and $b$ is a positive constant.

2.2.1. MFAC Design. For the one-step-ahead controller [44], the control goal is to seek a control input sequence $\mathbf{u}(k)$ that brings $y(k+1)$ to $y_{d}$, where $y_{d}$ is the desired system output signal. In general, the weighted one-step-ahead controller may lead to steady-state tracking error [16]. So the following control input criterion function is used to design the control law:

$$
J(\mathbf{u}(k))=\left|y_{d}-y_{1}(k+1)\right|^{2}+\lambda\|\Delta \mathbf{u}(k)\|^{2},
$$

where $\lambda>0$ is a weighting constant.

Substituting the dynamic linearization form

$$
y_{1}(k+1)=y(k)+\varphi^{T}(k) \Delta \mathbf{u}(k)
$$


into (6), as well as letting $\partial J / \partial \mathbf{u}(k)$ be zero, gives

$$
\mathbf{u}(k)=\mathbf{u}(k-1)+\frac{\rho_{1} \varphi(k)\left(y_{d}-y(k)\right)}{\lambda+\|\boldsymbol{\varphi}(k)\|^{2}},
$$

where $\rho_{1} \in(0,1]$ is a step-size constant that is added to make (8) more general and $\varphi(k)$ is PPD vector.

Because PPD vector $\varphi(k)$ is unknown, it needs to be estimated. Define criterion function for the unknown PPD vector as follows:

$$
\begin{gathered}
J(\widehat{\boldsymbol{\varphi}}(k))=\left|\Delta y(k)-\widehat{\boldsymbol{\varphi}}^{T}(k) \Delta \mathbf{u}(k-1)\right|^{2} \\
+\mu\|\Delta \widehat{\boldsymbol{\varphi}}(k)\|^{2},
\end{gathered}
$$

where $\mu>0$ is a weighting factor and $\widehat{\varphi}(k)$ is the estimation of $\varphi(k)$.

Using optimal condition $\partial J / \partial \widehat{\varphi}(k)=0$ then gives

$$
\begin{aligned}
\widehat{\boldsymbol{\varphi}}(k) & \\
= & \widehat{\boldsymbol{\varphi}}(k-1) \\
& +\frac{\eta \Delta \mathbf{u}(k-1)\left(\Delta y(k)-\widehat{\boldsymbol{\varphi}}^{T}(k-1) \Delta \mathbf{u}(k-1)\right)}{\mu+\|\Delta \mathbf{u}(k-1)\|^{2}},
\end{aligned}
$$

where $\eta \in(0,2)$ is a step-size constant.

Defining the tracking error $e(k)=y_{d}-y(k)$ and combining PPD estimation and input control law, MFAC scheme is designed as follows:

$$
\begin{aligned}
\widehat{\varphi}(k) & \\
= & \widehat{\boldsymbol{\varphi}}(k-1) \\
& +\frac{\eta \Delta \mathbf{u}(k-1)\left(\Delta y(k)-\widehat{\boldsymbol{\varphi}}^{T}(k-1) \Delta \mathbf{u}(k-1)\right)}{\mu+\|\Delta \mathbf{u}(k-1)\|^{2}}, \\
\mathbf{u}(k) & =\mathbf{u}(k-1)+\frac{\rho_{1} \widehat{\boldsymbol{\varphi}}(k)}{\lambda+\|\widehat{\boldsymbol{\varphi}}(k)\|^{2}} e(k) .
\end{aligned}
$$

2.2.2. DDOILC Design. The control objective is to find an appropriate control input sequence $\mathbf{u}(k)$, such that the system output $y(k)$ follows $y_{d}$. Consider the control input criterion function as

$$
J(\mathbf{u}(k))=\left|y_{d}-y_{2}(k)\right|^{2}+\lambda\|\Delta \mathbf{u}(k)\|^{2} .
$$

Substituting the dynamic linearization equation

$$
y_{2}(k)=y(k-1)+\boldsymbol{\varphi}^{T}(k) \Delta \mathbf{u}(k)
$$

into (12) and the optimal condition leads to

$$
\mathbf{u}(k)=\mathbf{u}(k-1)+\frac{\rho_{2} \boldsymbol{\varphi}(k)}{\lambda+\|\boldsymbol{\varphi}(k)\|^{2}} e(k-1),
$$

where $\rho_{2} \in(0,1]$ determines the step size and $e(k-1)=$ $y_{d}-y(k-1)$.
Let $\widehat{\varphi}(k)$ denote its estimation at $k$ th iteration. Then define the criterion function as

$$
\begin{gathered}
J(\widehat{\boldsymbol{\varphi}}(k))=\left|\Delta y(k-1)-\widehat{\boldsymbol{\varphi}}^{T}(k) \Delta \mathbf{u}(k-1)\right|^{2} \\
+\mu\|\Delta \widehat{\boldsymbol{\varphi}}(k)\|^{2} .
\end{gathered}
$$

According to optimal condition, the iterative updating law of estimate $\widehat{\boldsymbol{\varphi}}(k)$ is developed as

$$
\begin{aligned}
& \widehat{\varphi}(k)=\widehat{\varphi}(k-1) \\
& +\frac{\eta \Delta \mathbf{u}(k-1)\left(\Delta y(k-1)-\widehat{\varphi}^{T}(k-1) \Delta \mathbf{u}(k-1)\right)}{\mu+\|\Delta \mathbf{u}(k-1)\|^{2}},
\end{aligned}
$$

where $\mu>0,0<\eta<2$.

The DDOILC scheme is designed as follows:

$$
\begin{aligned}
\widehat{\varphi}(k) & =\widehat{\varphi}(k-1) \\
+ & \frac{\eta \Delta \mathbf{u}(k-1)\left(\Delta y(k-1)-\widehat{\boldsymbol{\varphi}}^{T}(k-1) \Delta \mathbf{u}(k-1)\right)}{\mu+\|\Delta \mathbf{u}(k-1)\|^{2}}, \\
\mathbf{u}(k) & =\mathbf{u}(k-1)+\frac{\rho_{2} \widehat{\boldsymbol{\varphi}}(k)}{\lambda+\|\widehat{\boldsymbol{\varphi}}(k)\|^{2}} e(k-1) .
\end{aligned}
$$

\section{Theoretical Analysis of MFAC and DDOILC and Simulations in SFBGP}

3.1. Theoretical Analysis of MFAC and DDOILC. The controller design procedures of MFAC and DDOILC have been introduced in previous sections. Although they have the same systemic framework and similar expression form of controller, there are still differences between them, which makes them two different methods. Comparing the controllers of MFAC and DDOILC, the main differences can be listed as follows:

(1) $\Delta y(k)$ and $\Delta y(k-1)$ are, respectively, used in PPD iterative updating laws.

(2) $e(k)$ and $e(k-1)$ are separately utilized in control input iterative learning laws.

The expressions of $\Delta y(k), \Delta y(k-1), e(k)$, and $e(k-1)$ are listed as follows:

$$
\text { MFAC: }\left\{\begin{array}{l}
\Delta y(k)=y(k)-y(k-1) \\
e(k)=y_{d}-y(k),
\end{array}\right.
$$

$$
\text { DDOILC: }\left\{\begin{array}{l}
\Delta y(k-1)=y(k-1)-y(k-2) \\
e(k-1)=y_{d}-y(k-1) .
\end{array}\right.
$$

Obviously, the main differences between MFAC and DDOILC can be described as follows: system output $y(k)$ at $k$ th iteration is used in MFAC but is not used in DDOILC. In practical application, all the system outputs used in DDOILC, $y(k-1)$ and $y(k-2)$, are the actual values at $(k-1)$ th and $(k-$ $2)$ th iterations. In MFAC, however, the actual value of system 


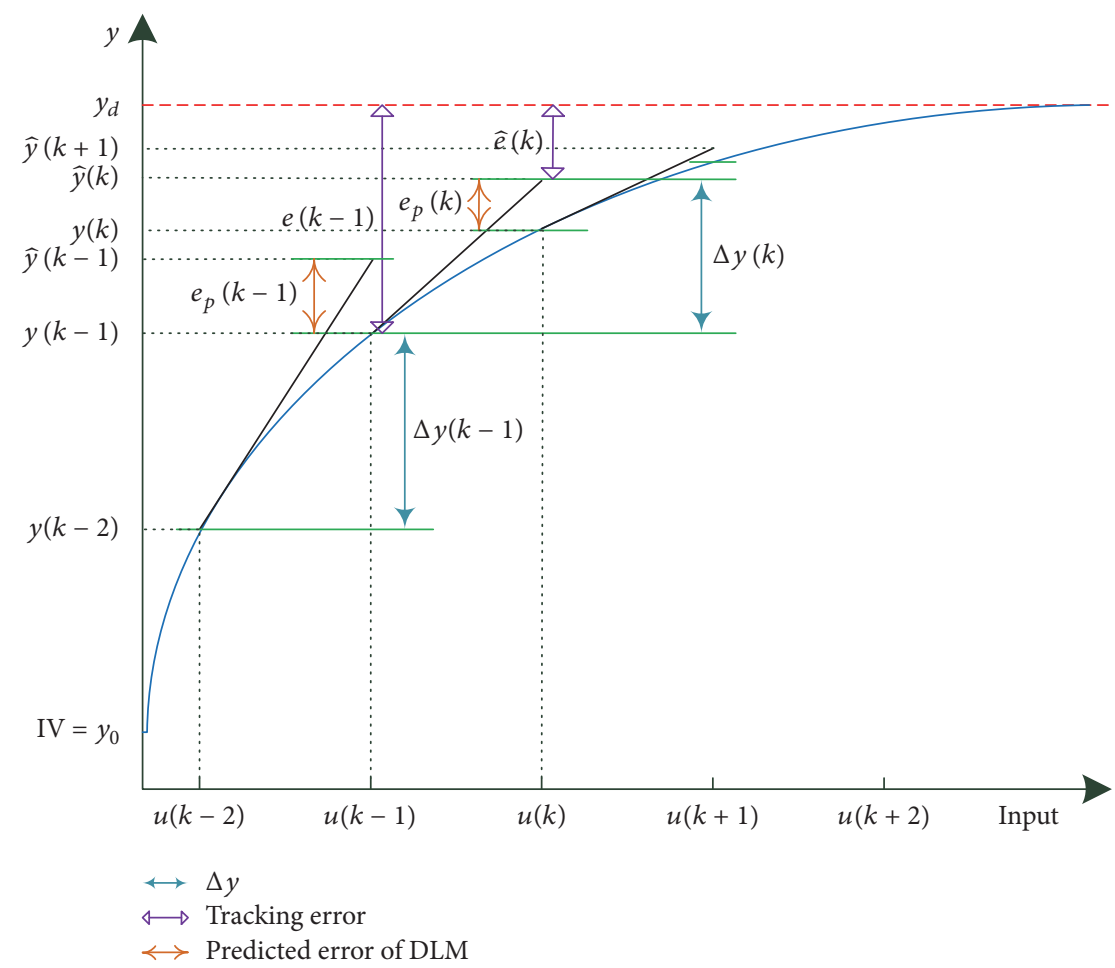

FIGURE 2: The variable relations during control process of MFAC and DDOILC.

output $y(k)$ is obtained only after the calculation of $\mathbf{u}(k)$. So $y(k)$ does not have actual value when computing $\mathbf{u}(k)$, and a predicted system output $\hat{y}(k)$ will be used to replace $y(k)$ in practical application of MFAC. $\widehat{y}(k)$ is estimated by the following DLM:

$$
\hat{y}(k)=y(k-1)+\varphi^{T}(k-1) \Delta \mathbf{u}(k-1),
$$

and $e(k)$ is replaced by $\widehat{e}(k)=y_{d}-\widehat{y}(k)$.

Figure 2 gives the variable relations during control process of MFAC and DDOILC. In terms of a single control process curve, when system output $y$ is greatly different from $y_{d}$, that is, the tracking error $e$ is relatively great, $e(k-1)$ is much larger than $\hat{e}(k)$ and DDOILC will achieve a bigger updating step size for control input, which gives DDOILC a faster convergence speed. As $y$ keeps getting closer to $y_{d}$, it is found that DLM prediction error $e_{p}$ is getting smaller and smaller until $e_{p}=0$. The difference between $e(k-1)$ and $\widehat{e}(k)$ will also be smaller and smaller. DDOILC will not only lose its advantage, but also perform worse than MFAC when $y$ is very close to $y_{d}$, because a larger updating step size for control input will bring difficulty in convergence, while, at this time, MFAC will achieve a fast and stable control effect due to the improvement of prediction accuracy of DLM. From another point of view, if IV of system output $y_{0}$ is far away from $y_{d}$, the initial tracking error and DLM prediction error will be both at a high level and DDOILC will perform better than MFAC because a greater updating step size for control input is necessary to give a faster convergence speed. But as $y_{0}$ keeps getting closer to $y_{d}, e_{p}$ will gradually decrease and MFAC will gradually show its advantage. Therefore, the conclusions obtained from theoretical analysis can be summarized as follows:

(1) When tracking error is large and $y_{0}$ is relatively far away from $y_{d}$, DDOILC will have a faster convergence speed and a better control performance.

(2) When tracking error gradually decreases and $y_{0}$ gradually gets closer to $y_{d}$, MFAC will gradually reveal its advantage and achieve better control effect.

3.2. Simulation Verification. To validate conclusions obtained from theoretical analysis, simulation verification in SFBGP is conducted. In this simulation, particle quality to be controlled is APS, and the desired quality is $y_{d}=120 \mu \mathrm{m}$. The viscosity of binder is treated as material attribute to be changed, and then a series of IVs $\left(y_{0}\right)$ are constructed by adjusting viscosity of binder while keeping the operating condition unchanged. Four sets of IVs are selected as representatives in this work, and the detailed establishing information of them is shown in Table 1. Besides, the parameter settings of MFAC and DDOILC for different IVs are listed in Table 2.

For different IVs, we conduct particle quality control in SFBGP based on MFAC and DDOILC, and the control performances are plotted in Figure 3. Moreover, DLM prediction error profiles of MFAC are shown in Figure 4. Then the explanation and analysis of simulation results will be given. As is shown in Figure 3(a), DDOILC performs better than MFAC when $y_{0}=60$; that is, $y_{0}$ is relatively far away from $y_{d}$. When $y_{0}=70$, as is described in Figure 3(b), DDOILC has a faster convergence speed when APS is controlled from $70 \mu \mathrm{m}$ to about $110 \mu \mathrm{m}$, but MFAC achieves a relatively better 


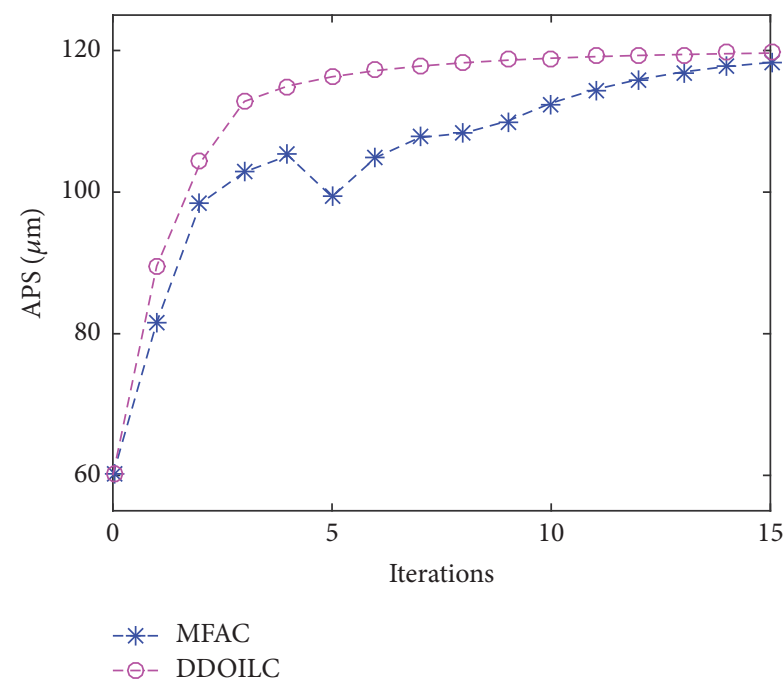

(a) $y_{0}=60$

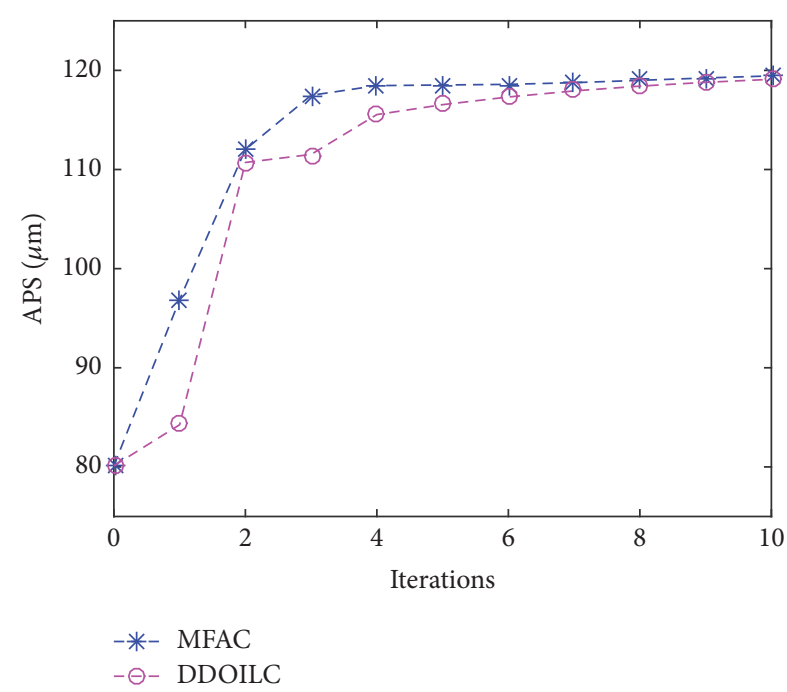

(c) $y_{0}=80$

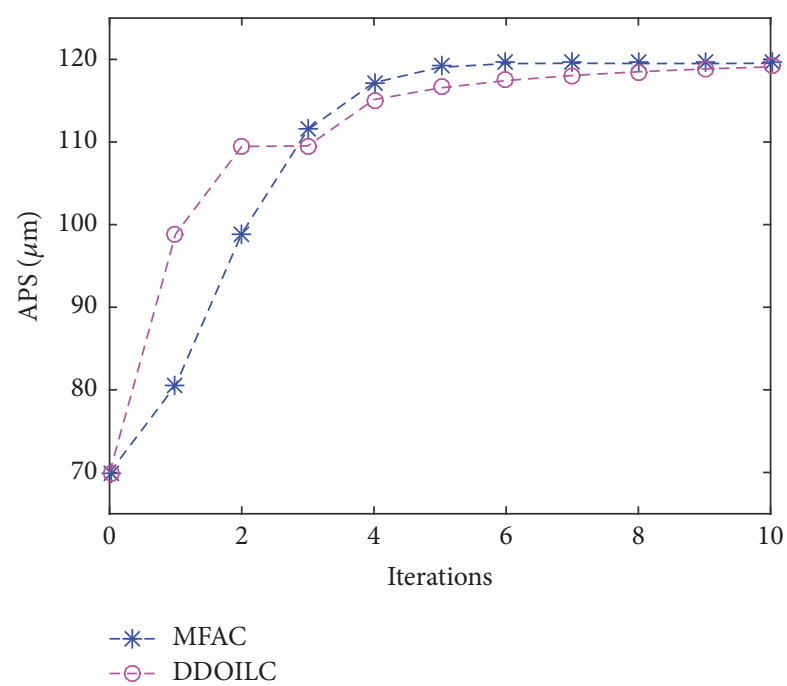

(b) $y_{0}=70$

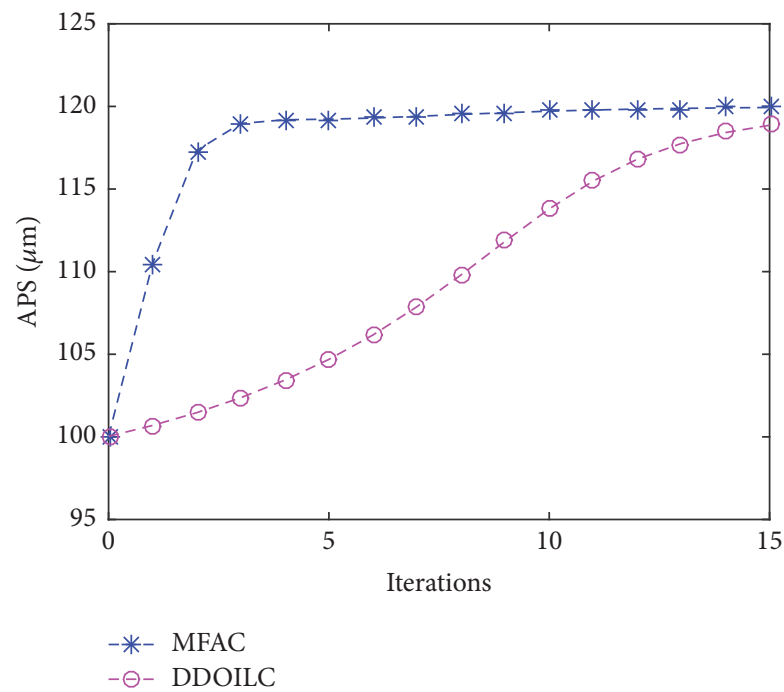

(d) $y_{0}=100$

Figure 3: The control profiles of APS in SFBGP based on MFAC and DDOILC.

TABLE 1: The detailed establishing information of representative IVs.

\begin{tabular}{lllll}
\hline Info & \multicolumn{4}{c}{ IV } \\
& $y_{0}=60$ & $y_{0}=70$ & $y_{0}=80$ & $y_{0}=100$ \\
\hline $\begin{array}{l}\text { Operating } \\
\text { conditions } \\
(\mathrm{g} / \mathrm{s})\end{array}$ & First stage: 0.3 ; second stage: 0.1 ; third stage: 0.15 \\
$\begin{array}{l}\text { Viscosity of } \\
\text { binder }(\mathrm{Pa} \cdot \mathrm{s})\end{array}$ & 0.0214 & 0.0250 & 0.0304 & 0.0625 \\
\hline
\end{tabular}

control performance when it is controlled from $110 \mu \mathrm{m}$ to $y_{d}$, which indicates that both DDOILC and MFAC have certain advantages in such case. However, from Figure 3(c), it can be seen that MFAC is slightly superior to DDOILC in control effect when $y_{0}=80$, and this just manifests the advantage of MFAC. As $y_{0}$ gets closer and closer to $y_{d}$, such as $y_{0}=100$
TABLE 2: The parameter settings of MFAC and DDOILC for different IVs.

\begin{tabular}{lccccc}
\hline IV & \multicolumn{5}{c}{ Parameter } \\
& $\rho_{1}$ & $\rho_{2}$ & $\lambda$ & $\eta$ & $\mu$ \\
\hline$y_{0}=60$ & 0.0505 & 0.0712 & & & \\
$y_{0}=70$ & 0.0047 & 0.0503 & 0.0010 & 0.0750 & 1.0000 \\
$y_{0}=80$ & 0.0032 & 0.0199 & & & \\
$y_{0}=100$ & 0.0020 & $1.49 E-4$ & & & \\
\hline
\end{tabular}

plotted in Figure 3(d), MFAC has a huge advantage compared with DDOILC. It is obvious that the simulation results have verified the conclusions obtained from theoretical analysis. The reason for increasing superiority of MFAC is attributed to the smaller and smaller prediction error $e_{p}$ of DLM. As is shown in Figure 4, on the one hand, the curves of $e_{p}$ for 


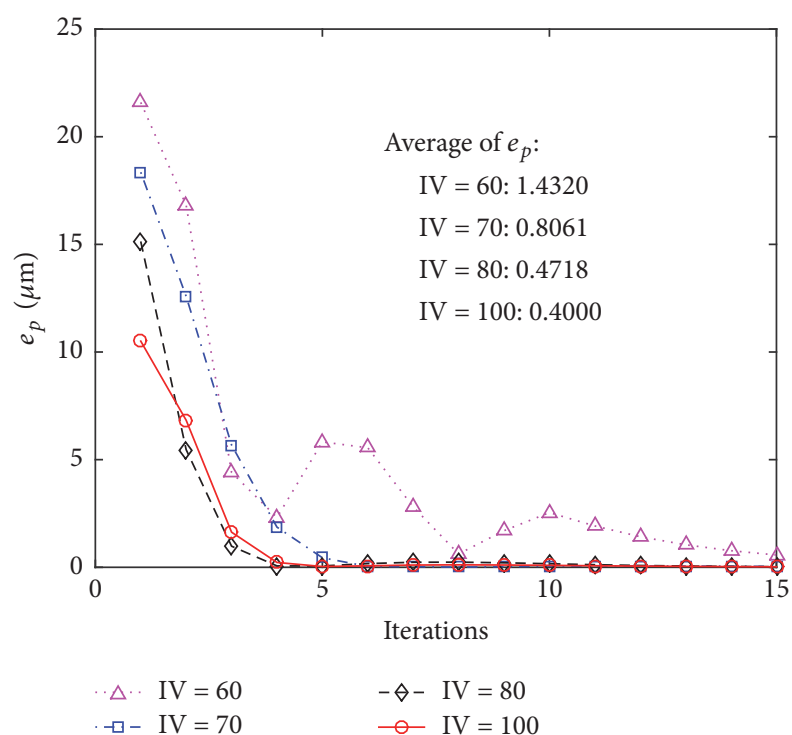

FIGURE 4: The DLM prediction error profiles of MFAC for different IVs.

different IVs all show a decreasing trend along with iteration until $e_{p}=0$ as APS keeps getting closer to $y_{d}$, which is in accordance with our obtained conclusion. On the other hand, for different IVs, $e_{p}$ will also decrease with the increase of IV, which is proved by comparing the curves of $e_{p}$ under different IVs. To further illustrate this point, the averages of $e_{p}$ for different IVs are computed and then listed in Figure 4, and the decreasing averages of $e_{p}$ along with the increase of IV also validate the rationality of our analysis.

\section{The Proposed Hybrid DDC Approach and Simulation Study in SFBGP}

Through theoretical analysis and simulation study, we have proved that MFAC and DDOILC both have strengths in particle quality control under different IVs. In real-world applications, the same operating condition may give entirely different IVs due to the changes in material properties, which brings great difficulties in the selection of control methods. Thus, the uncertainties of IV have to be considered during control application. In order to achieve favourable overall control effect, it is demanded that the DDC approaches must accommodate various IVs. Therefore, we try to combine advantages of MFAC and DDOILC to present a hybrid approach that is named DDMFAC. The basic idea is that the features of MFAC and DDOILC are both reflected in the criterion functions when designing controller of DDMFAC. The expected features of hybrid approach can be summarized as the following three points:

(1) When tracking error is large, DDMFAC manifests the feature of DDOILC to acquire a faster convergence speed.

(2) When tracking error is small enough, DDMFAC has the ability of MFAC to achieve a fast and stable control effect.
(3) DDMFAC is able to achieve better control performance for different IVs.

Through such combination, the shortcomings of MFAC and DDOILC will be complemented and their respective advantages will be highlighted in DDMFAC. Next we describe the controller design for DDMFAC in Section 4.1.

4.1. DDMFAC Design. Consider the following control input criterion function:

$$
\begin{aligned}
J(\mathbf{u}(k))= & \alpha_{M}\left|y_{d}-y_{1}(k+1)\right|^{2}+\alpha_{D}\left|y_{d}-y_{2}(k)\right|^{2} \\
& +\lambda_{1}\|\Delta \mathbf{u}(k)\|^{2},
\end{aligned}
$$

where $\left|y_{d}-y_{1}(k+1)\right|^{2}$ and $\left|y_{d}-y_{2}(k)\right|^{2}$, respectively, come from MFAC and DDOILC and $\alpha_{M}, \alpha_{D}$ are the respective weighted factors and $\alpha_{M}+\alpha_{D} \neq 0$.

Substituting (7) and (13) into (20) gives

$$
\begin{aligned}
J(\mathbf{u}(k))= & \alpha_{M}\left\|e(k)-\varphi^{T}(k) \Delta \mathbf{u}(k)\right\|^{2} \\
& +\alpha_{D}\left\|e(k-1)-\varphi^{T}(k) \Delta \mathbf{u}(k)\right\|^{2} \\
& +\lambda_{1}\|\Delta \mathbf{u}(k)\|^{2} .
\end{aligned}
$$

Using optimal condition $\partial J / \partial \mathbf{u}(k)=0$ then gives

$$
\Delta \mathbf{u}(k)=\frac{\boldsymbol{\varphi}(k)\left(\alpha_{M} e(k)+\alpha_{D} e(k-1)\right)}{\lambda_{1}+\left(\alpha_{M}+\alpha_{D}\right)\|\varphi(k)\|^{2}} .
$$

Dividing numerator and denominator by $\alpha_{M}+\alpha_{D}$ at the right hand of (22), we have

$$
\begin{aligned}
& \Delta \mathbf{u}(k) \\
& =\frac{\varphi(k)\left(\left(\alpha_{M} /\left(\alpha_{M}+\alpha_{D}\right)\right) e(k)+\left(\alpha_{D} /\left(\alpha_{M}+\alpha_{D}\right)\right) e(k-1)\right)}{\left(\lambda_{1} /\left(\alpha_{M}+\alpha_{D}\right)\right)+\|\varphi(k)\|^{2}} .
\end{aligned}
$$

Letting $\rho_{M}=\alpha_{M} /\left(\alpha_{M}+\alpha_{D}\right), \rho_{D}=\alpha_{D} /\left(\alpha_{M}+\alpha_{D}\right)$, and $\lambda=$ $\lambda_{1} /\left(\alpha_{M}+\alpha_{D}\right)$, then

$$
\mathbf{u}(k)=\mathbf{u}(k-1)+\frac{\boldsymbol{\varphi}(k)\left(\rho_{M} e(k)+\rho_{D} e(k-1)\right)}{\lambda+\|\boldsymbol{\varphi}(k)\|^{2}},
$$

where $\lambda>0$ is the weighted factor and $\rho_{M}, \rho_{D} \in[0,1]$ are the step-size factors.

Define the following PPD vector criterion function:

$$
\begin{aligned}
& J(\widehat{\boldsymbol{\varphi}}(k)) \\
& =\left\|\beta_{M} \Delta y(k)+\beta_{D} \Delta y(k-1)-\widehat{\boldsymbol{\varphi}}^{T}(k) \Delta \mathbf{u}(k-1)\right\|^{2} \\
& \quad+\mu\|\Delta \widehat{\boldsymbol{\varphi}}(k)\|^{2},
\end{aligned}
$$

where $\beta_{M}, \beta_{D} \in[0,1]$ are the weighted factors and $\beta_{M}+\beta_{D}=$ 1.

We rewrite (25) as

$$
\begin{aligned}
& J(\widehat{\boldsymbol{\varphi}}(k))=\mid \beta_{M} \Delta y(k)+\beta_{D} \Delta y(k-1) \\
& \quad-\widehat{\boldsymbol{\varphi}}^{T}(k-1) \Delta \mathbf{u}(k-1)-\left.\Delta \widehat{\boldsymbol{\varphi}}^{T}(k) \Delta \mathbf{u}(k-1)\right|^{2} \\
& \quad+\mu\|\Delta \widehat{\boldsymbol{\varphi}}(k)\|^{2} .
\end{aligned}
$$


Utilizing optimal condition $\partial J / \partial \widehat{\boldsymbol{\varphi}}(k)=0$, we have

$$
\widehat{\boldsymbol{\varphi}}(k)=\widehat{\varphi}(k-1)+\eta \frac{\Delta \mathbf{u}(k-1)\left(\beta_{M} \Delta y(k)+\beta_{D} \Delta y(k-1)-\widehat{\boldsymbol{\varphi}}^{T}(k-1) \Delta \mathbf{u}(k-1)\right)}{\mu+\|\Delta \mathbf{u}(k-1)\|^{2}} .
$$

Thus, the controller of DDMFAC is designed as follows:

$$
\begin{aligned}
& \widehat{\boldsymbol{\varphi}}(k)=\widehat{\boldsymbol{\varphi}}(k-1)+\eta \frac{\Delta \mathbf{u}(k-1)\left(\beta_{M} \Delta y(k)+\beta_{D} \Delta y(k-1)-\widehat{\boldsymbol{\varphi}}^{T}(k-1) \Delta \mathbf{u}(k-1)\right)}{\mu+\|\Delta \mathbf{u}(k-1)\|^{2}}, \\
& \mathbf{u}(k)=\mathbf{u}(k-1)+\frac{\boldsymbol{\varphi}(k)\left(\rho_{M} e(k)+\rho_{D} e(k-1)\right)}{\lambda+\|\boldsymbol{\varphi}(k)\|^{2}} .
\end{aligned}
$$

4.2. Stability and Convergence Analysis of DDMFAC

$$
\widetilde{\varphi}(k)=\varphi(k)-\widehat{\varphi}(k) .
$$

(1) The Boundedness of $\widehat{\varphi}(k)$. Define the parameter estimation error of $\varphi(k)$ as follows:

$$
\widetilde{\boldsymbol{\varphi}}(k)=\boldsymbol{\varphi}(k)-\widehat{\boldsymbol{\varphi}}(k-1)-\eta \frac{\Delta \mathbf{u}(k-1)\left[\beta_{M} \Delta y(k)+\beta_{D} \Delta y(k-1)-\widehat{\boldsymbol{\varphi}}^{T}(k-1) \Delta \mathbf{u}(k-1)\right]}{\mu+\|\Delta \mathbf{u}(k-1)\|^{2}} .
$$

Similarly, define $\widetilde{\boldsymbol{\varphi}}(k-1)=\boldsymbol{\varphi}(k-1)-\widehat{\boldsymbol{\varphi}}(k-1)$, and then

$$
\widehat{\varphi}(k-1)=\varphi(k-1)-\widetilde{\varphi}(k-1) .
$$

$$
\widetilde{\boldsymbol{\varphi}}(k)=\widetilde{\boldsymbol{\varphi}}(k-1)-\eta \frac{\Delta \mathbf{u}(k-1)\left[\beta_{M} \Delta y(k)+\beta_{D} \Delta y(k-1)-\widehat{\boldsymbol{\varphi}}^{T}(k-1) \Delta \mathbf{u}(k-1)\right]}{\mu+\|\Delta \mathbf{u}(k-1)\|^{2}}+\boldsymbol{\varphi}(k)-\boldsymbol{\varphi}(k-1) .
$$

According to (7) and (13), it can be obtained that

$$
\Delta y(k-1)=\varphi^{T}(k-1) \Delta \mathbf{u}(k-1) .
$$

$$
\Delta y(k)=\varphi^{T}(k-1) \Delta \mathbf{u}(k-1)
$$

$$
\begin{aligned}
\widetilde{\boldsymbol{\varphi}}(k) & =\widetilde{\boldsymbol{\varphi}}(k-1)-\eta \frac{\Delta \mathbf{u}(k-1)\left(\left(\beta_{M}+\beta_{D}\right) \boldsymbol{\varphi}^{T}(k-1) \Delta \mathbf{u}(k-1)-\widehat{\boldsymbol{\varphi}}^{T}(k-1) \Delta \mathbf{u}(k-1)\right)}{\mu+\|\Delta \mathbf{u}(k-1)\|^{2}}+\boldsymbol{\varphi}(k)-\boldsymbol{\varphi}(k-1) \\
& =\widetilde{\boldsymbol{\varphi}}(k-1)-\eta \frac{\Delta \mathbf{u}(k-1)\left((\boldsymbol{\varphi}(k-1)-\widehat{\boldsymbol{\varphi}}(k-1))^{T} \Delta \mathbf{u}(k-1)\right)}{\mu+\|\Delta \mathbf{u}(k-1)\|^{2}}+\boldsymbol{\varphi}(k)-\boldsymbol{\varphi}(k-1) \\
& =\widetilde{\boldsymbol{\varphi}}(k-1)-\eta \frac{\widetilde{\boldsymbol{\varphi}}(k-1)\|\Delta \mathbf{u}(k-1)\|^{2}}{\mu+\|\Delta \mathbf{u}(k-1)\|^{2}}+\boldsymbol{\varphi}(k)-\boldsymbol{\varphi}(k-1) \\
& =\widetilde{\boldsymbol{\varphi}}(k-1)\left(1-\eta \frac{\|\Delta \mathbf{u}(k-1)\|^{2}}{\mu+\|\Delta \mathbf{u}(k-1)\|^{2}}\right)+\boldsymbol{\varphi}(k)-\boldsymbol{\varphi}(k-1) .
\end{aligned}
$$


Because $|\varphi(k)| \leq b$ is bounded [16], $|\varphi(k)-\varphi(k-1)| \leq 2 b$. Taking the modulus of both sides of (35), we can obtain

$\|\widetilde{\varphi}(k)\|$

$$
\begin{gathered}
\leq\left\|\widetilde{\boldsymbol{\varphi}}(k-1)\left(1-\eta \frac{\|\Delta \mathbf{u}(k-1)\|^{2}}{\mu+\|\Delta \mathbf{u}(k-1)\|^{2}}\right)\right\|+2 b, \\
\left\|\widetilde{\boldsymbol{\varphi}}(k-1)\left(1-\eta \frac{\|\Delta \mathbf{u}(k-1)\|^{2}}{\mu+\|\Delta \mathbf{u}(k-1)\|^{2}}\right)\right\|^{2} \\
=\|\tilde{\boldsymbol{\varphi}}(k-1)\|^{2}+\left(-2+\eta \frac{\|\Delta \mathbf{u}(k-1)\|^{2}}{\mu+\|\Delta \mathbf{u}(k-1)\|^{2}}\right) \\
\quad \times \eta \frac{\|\widetilde{\boldsymbol{\varphi}}(k-1) \Delta \mathbf{u}(k-1)\|^{2}}{\mu+\|\Delta \mathbf{u}(k-1)\|^{2}} .
\end{gathered}
$$

Given that $0<\eta<2, \mu>0$,

$$
\left(-2+\eta \frac{\|\Delta \mathbf{u}(k-1)\|^{2}}{\mu+\|\Delta \mathbf{u}(k-1)\|^{2}}\right)<0 .
$$

Synthesizing (37) and (38), we have

$$
\begin{aligned}
& \left\|\widetilde{\boldsymbol{\varphi}}(k-1)\left(1-\eta \frac{\|\Delta \mathbf{u}(k-1)\|^{2}}{\mu+\|\Delta \mathbf{u}(k-1)\|^{2}}\right)\right\|^{2} \\
& \quad \leq\|\tilde{\boldsymbol{\varphi}}(k-1)\|^{2} .
\end{aligned}
$$

According to (36) and (39), there is obviously a positive number $d_{1} \in(0,1)$, which makes

$$
\begin{aligned}
\|\widetilde{\boldsymbol{\varphi}}(k)\| & \leq d_{1}\|\widetilde{\boldsymbol{\varphi}}(k-1)\|+2 b \leq \cdots \\
& \leq d_{1}^{k}\|\widetilde{\boldsymbol{\varphi}}(0)\|+\frac{2 b\left(1-d_{1}^{k}\right)}{1-d_{1}} .
\end{aligned}
$$

Therefore, $\widehat{\varphi}(k)$ is bounded.

(2) The Convergence of Tracking Error e $(k)$. Tracking error $e(k)$ is derived as

$$
\begin{aligned}
e(k)= & y_{d}-y(k)=y_{d}-y(k-1)-\varphi^{T}(k) \Delta \mathbf{u}(k) \\
= & e(k-1)-\frac{\|\varphi(k)\|^{2}\left(\rho_{M} e(k)+\rho_{D} e(k-1)\right)}{\left(\lambda+\|\varphi(k)\|^{2}\right)} \\
= & \left(1-\frac{\rho_{D}\|\varphi(k)\|^{2}}{\lambda+\|\varphi(k)\|^{2}}\right) e(k-1) \\
& -\frac{\rho_{M}\|\varphi(k)\|^{2}}{\lambda+\|\varphi(k)\|^{2}} e(k) .
\end{aligned}
$$

Transposition gives

$$
e(k)=\left[1-\frac{\left(\rho_{M}+\rho_{D}\right)\|\varphi(k)\|^{2}}{\lambda+\left(1+\rho_{M}\right)\|\varphi(k)\|^{2}}\right] e(k-1) .
$$

So as long as appropriate parameters $\rho_{M}, \rho_{D}$, and $\lambda$ are selected, there must be a positive number $d_{2} \in(0,1)$ that makes

$$
0<\left\|1-\frac{\left(\rho_{M}+\rho_{D}\right)\|\varphi(k)\|^{2}}{\lambda+\left(1+\rho_{M}\right)\|\varphi(k)\|^{2}}\right\| \leq d_{2}<1 .
$$

By (42) and (43), it can be obtained that

$$
\begin{aligned}
\|e(k)\| & \leq\left\|\left(1-\frac{\left(\rho_{M}+\rho_{D}\right)\|\varphi(k)\|^{2}}{\lambda+\left(1+\rho_{M}\right)\|\varphi(k)\|^{2}}\right) e(k-1)\right\| \\
& \leq d_{2}\|e(k-1)\| \leq \cdots \leq d_{2}^{k}\|e(0)\| .
\end{aligned}
$$

Therefore, $\lim _{k \rightarrow \infty} e(k)=0$; that is, $e(k)$ is proved to converge to zero.

(3) The Boundedness of System Input and Output. By referring to $a^{2}+b^{2} \geq 2 a b$, the relational expression can be obtained as follows:

$$
\left\|\frac{\varphi(k)}{\lambda+\|\varphi(k)\|^{2}}\right\| \leq\left\|\frac{\varphi(k)}{2 \sqrt{\lambda}\|\varphi(k)\|}\right\|=\frac{1}{2 \sqrt{\lambda}} .
$$

And we have

$$
\begin{aligned}
\mathbf{u}(k)= & \mathbf{u}(k)-\mathbf{u}(k-1)+\mathbf{u}(k-1)-\cdots+\mathbf{u}(1) \\
& -\mathbf{u}(0)+\mathbf{u}(0)=\mathbf{u}(0)+\sum_{j=1}^{k} \mathbf{u}(j) .
\end{aligned}
$$

Combining (24), (44), (45), and (46), as well as utilizing Schwartz's inequality, gives

$$
\begin{aligned}
\|\mathbf{u}(k)\| \leq & \|\mathbf{u}(0)\|+\sum_{j=1}^{k}\|\Delta \mathbf{u}(j)\| \\
\leq & \|\mathbf{u}(0)\|+\frac{1}{2 \sqrt{\lambda}} \sum_{j=1}^{k}\left\|\rho_{D} e(j-1)+\rho_{M} e(j)\right\| \\
\leq & \|\mathbf{u}(0)\|+\frac{\rho_{D}}{2 \sqrt{\lambda}} \sum_{j=1}^{k} d_{2}^{j-1}\|e(0)\| \\
& +\frac{\rho_{M}}{2 \sqrt{\lambda}} \sum_{j=1}^{k} d_{2}^{j}\|e(0)\| \\
\leq & \|\mathbf{u}(0)\|+\frac{\rho_{D}}{2 \sqrt{\lambda}} \frac{1-d_{2}^{j-1}}{1-d_{2}}\|e(0)\| \\
& +\frac{\rho_{M}}{2 \sqrt{\lambda}} \frac{d_{2}\left(1-d_{2}^{j}\right)}{1-d_{2}}\|e(0)\| \\
\leq & \|\mathbf{u}(0)\|+\frac{\rho_{D}}{\left(1-d_{2}\right) 2 \sqrt{\lambda}}\|e(0)\|
\end{aligned}
$$


TABLE 3: The parameter settings of DDMFAC for different IVs.

\begin{tabular}{lccccccc}
\hline IV & & \multicolumn{3}{c}{ Parameter } & $\lambda$ & $\mu$ \\
& $\rho_{M}$ & $\rho_{D}$ & $\beta_{M}$ & $\beta_{D}$ & $\lambda$ & & \\
\hline$y_{0}=60$ & 0.0275 & 0.0275 & 0.5000 & 0.5000 & & & \\
$y_{0}=70$ & $5.0 E-4$ & $5.0 E-4$ & 0.5000 & 0.5000 & 0.0010 & 0.0750 & \\
$y_{0}=80$ & $4.6 E-4$ & $4.6 E-4$ & 0.5000 & 0.5000 & & & \\
$y_{0}=100$ & $1.8 E-4$ & $1.8 E-4$ & 0.5000 & 0.5000 & & & \\
\hline
\end{tabular}

$$
\begin{gathered}
+\frac{d_{2} \rho_{M}}{\left(1-d_{2}\right) 2 \sqrt{\lambda}}\|e(0)\| \\
\leq\|\mathbf{u}(0)\|+\frac{\rho_{D}+d_{2} \rho_{M}}{\left(1-d_{2}\right) 2 \sqrt{\lambda}}\|e(0)\| .
\end{gathered}
$$

Because the initial tracking error $e(0)$ and initial input $\mathbf{u}(0)$ are given bounded, the system input $\mathbf{u}(k)$ is bounded according to (47). Besides, due to the boundedness of $y_{d}$ and $e(k)$, the system output $y(k)$ is obviously bounded.

4.3. Fuzzy DDMFAC. The controller design and stability and convergence analysis for DDMFAC have been, respectively, discussed in detail in the above sections. Although the designed controller has the characters of both MFAC and DDOILC, weighted factors $\rho_{M}, \rho_{D}, \beta_{M}$, and $\beta_{D}$ are all constant throughout control process. DDMFAC is incapable of adaptively selecting the dominance of MFAC or DDOILC to duly show the expected features in each stage of control process. The aforementioned features of DDMFAC we expect cannot be reflected in this case. Such DDMFAC approach with fixed weighting factors has no advantage compared with MFAC or DDOILC, which appeals to a reasonable and adaptive adjustment strategy for $\rho_{M}, \rho_{D}, \beta_{M}$, and $\beta_{D}$. Therefore, the adaptive adjustment strategy based on fuzzy logic for weighting factors is considered because its advantageous performance in parameter adjustment has been proven in our previous work [45]. The formulation of fuzzy rules offers us the opportunity to adjust weighting factors in the way we expected. Such DDMFAC approach with fuzzy adaptive adjustment for parameters is named FDDMFAC in this paper. Concretely, the fuzzy rules can be formulated by referring to the expected features of DDMFAC. The input variables of fuzzy rules are the absolute values of tracking error $e(k-1)$ and DLM prediction error $e_{p}(k-1)$ of MFAC, and the output variables are $\rho_{M}, \rho_{D}$, and $\beta_{D}$. Because of the relationship between $\beta_{M}$ and $\beta_{D}$, there is no need to use $\beta_{M}$ as an output and it is calculated by $\beta_{M}=1-\beta_{D}$. The fuzzy rules can be detailedly described as follows:

(1) If $|e(k-1)|$ and $\left|e_{p}(k-1)\right|$ are great, then the dominance of DDOILC is preferred and that of MFAC is restrained in DDMFAC. So $\rho_{D}$ and $\beta_{D}$ have relatively large values, and $\rho_{M}$ is a relatively small number.

(2) $\rho_{D}$ and $\beta_{D}$ will show a decreasing trend with the gradual decrease of $|e(k-1)|$ and $\left|e_{p}(k-1)\right|$, while $\rho_{M}$ will present an increasing trend.
4.4. Simulations and Result Analysis. The proposed DDMFAC approach is firstly applied to the particle quality control in SFBGP, and then its control effect is compared with MFAC and DDOILC under different IVs. In the simulation, just like MFAC, $y(k)$ is replaced by a predicted value $\hat{y}(k)$ that is estimated using (19). See Table 3 for parameter settings of DDMFAC. It is not hard to find $\rho_{M}=\rho_{D}$ and $\beta_{M}=\beta_{D}$ for a certain IV, which means that MFAC and DDOILC are set to have the same dominance in DDMFAC. By the way, MFAC and DDOILC can be regarded as the extreme cases of DDMFAC described as follows:

$$
\begin{aligned}
\text { MFAC: } & \rho_{M}=\beta_{M}=1, \\
& \rho_{D}=\beta_{D}=0 ; \\
\text { DDOILC: } & \rho_{M}=\beta_{M}=0, \\
& \rho_{D}=\beta_{D}=1 .
\end{aligned}
$$

The control effects of DDMFAC with the parameter settings in Table 3, together with control curves of MFAC and DDOILC, are plotted in Figure 5. The simulation results actually present the control effects of DDMFAC with three sets of parameter settings. From simulation, it is indicated that all three approaches have gained some certain advantages under different IV conditions. Concretely, as is shown in Figure 5(a), DDMFAC has advantage in control performance compared with DDOILC and MFAC when $y_{0}=60$, but MFAC has absolute superiority when $y_{0}=100$ plotted in Figure 5(d). In addition, from the simulation results in Figures 5(b) and 5(c), it can be seen that DDOILC and MFAC have achieved certain but not absolute advantages, respectively. This demonstrates that parameter setting has important influence on the control effect of DDMFAC. Therefore, the way to achieve satisfactory control effect is to adaptively adjust weighted parameters of DDMFAC, instead of keeping them constant.

Then, the idea of fuzzy adjustment is introduced to adjust the weights $\rho_{M}, \rho_{D}$, and $\beta_{D}$. The fuzzy adjustment rules of three weights are, respectively, shown in Tables 4 and 5 . The respective membership functions when $y_{0}=60$ are shown in Figure 6, and membership functions for the other IVs are no longer displayed. Please see Figure 7 for control effect of FDDMFAC and comparisons with MFAC and DDOILC under different IVs. Obviously, DDMFAC approach with fuzzy adjustment for parameters, that is, FDDMFAC, has the best performance in particle quality control for all IVs. This 
TABLE 4: The fuzzy rules of $\rho_{M}$.

\begin{tabular}{lcccccc}
\hline$\rho_{M}$ & & & & & \\
$|e(k-1)|$ & $\mathrm{NB}$ & $\mathrm{NM}$ & $\mathrm{NS}$ & $\mathrm{Z}$ & $\mathrm{PS}$ & $\mathrm{PM}$ \\
\hline $\mathrm{NB}$ & $\mathrm{PB}$ & $\mathrm{PB}$ & $\mathrm{PB}$ & $\mathrm{PB}$ & $\mathrm{PB}$ & $\mathrm{PB}$ \\
$\mathrm{NM}$ & $\mathrm{PB}$ & $\mathrm{PM}$ & $\mathrm{PM}$ & $\mathrm{PM}$ & $\mathrm{PM}$ & $\mathrm{PB}$ \\
$\mathrm{NS}$ & $\mathrm{PM}$ & $\mathrm{PM}$ & $\mathrm{PM}$ & $\mathrm{PS}$ & $\mathrm{PS}$ & $\mathrm{P}$ \\
$\mathrm{Z}$ & $\mathrm{Z}$ & $\mathrm{Z}$ & $\mathrm{Z}$ & $\mathrm{NS}$ & $\mathrm{Z}$ \\
$\mathrm{PS}$ & $\mathrm{NS}$ & $\mathrm{NS}$ & $\mathrm{NM}$ & $\mathrm{NM}$ & $\mathrm{NB}$ & $\mathrm{NS}$ \\
$\mathrm{PM}$ & $\mathrm{NM}$ & $\mathrm{NM}$ & $\mathrm{NM}$ & $\mathrm{NB}$ & $\mathrm{NB}$ & $\mathrm{NB}$ \\
$\mathrm{PB}$ & $\mathrm{NB}$ & $\mathrm{NB}$ & $\mathrm{NB}$ & $\mathrm{NB}$ & $\mathrm{NB}$ & $\mathrm{NB}$ \\
\hline
\end{tabular}

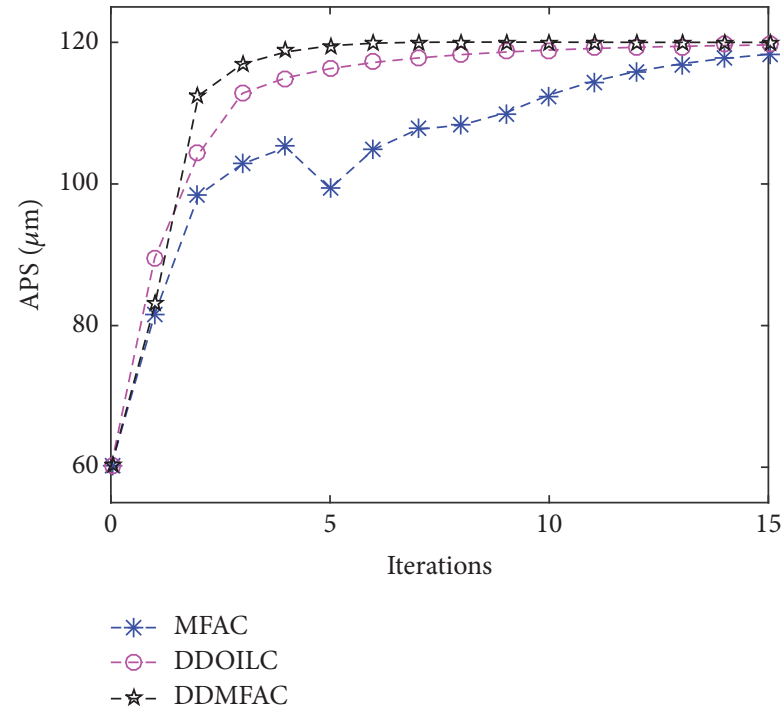

(a) $y_{0}=60$

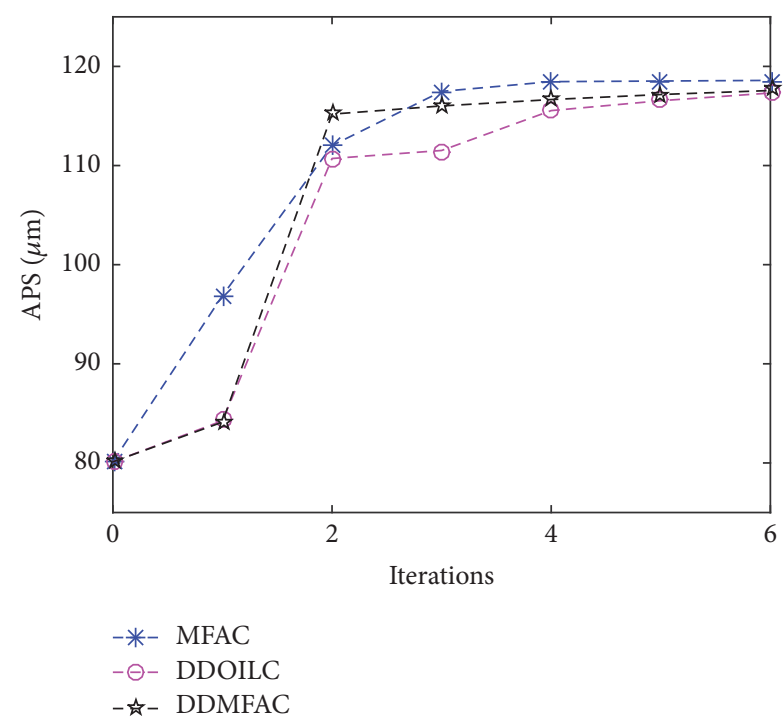

(c) $y_{0}=80$

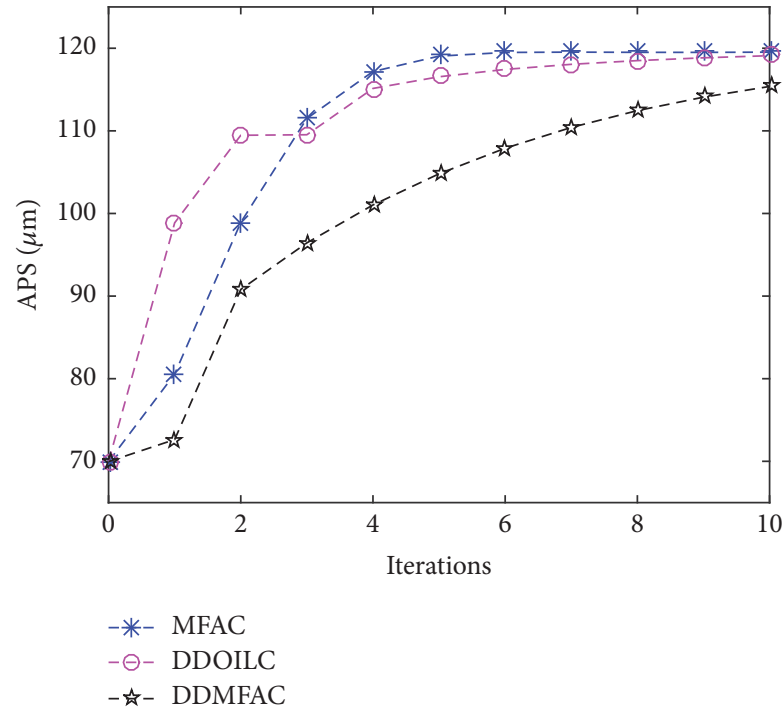

(b) $y_{0}=70$

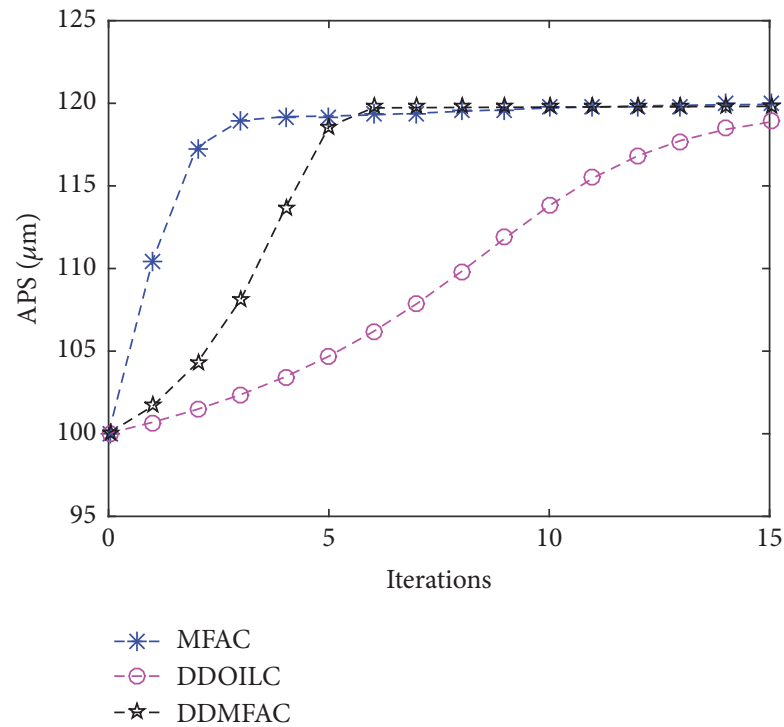

(d) $y_{0}=100$

Figure 5: The comparison of control effects of MFAC, DDOILC, and DDMFAC. 

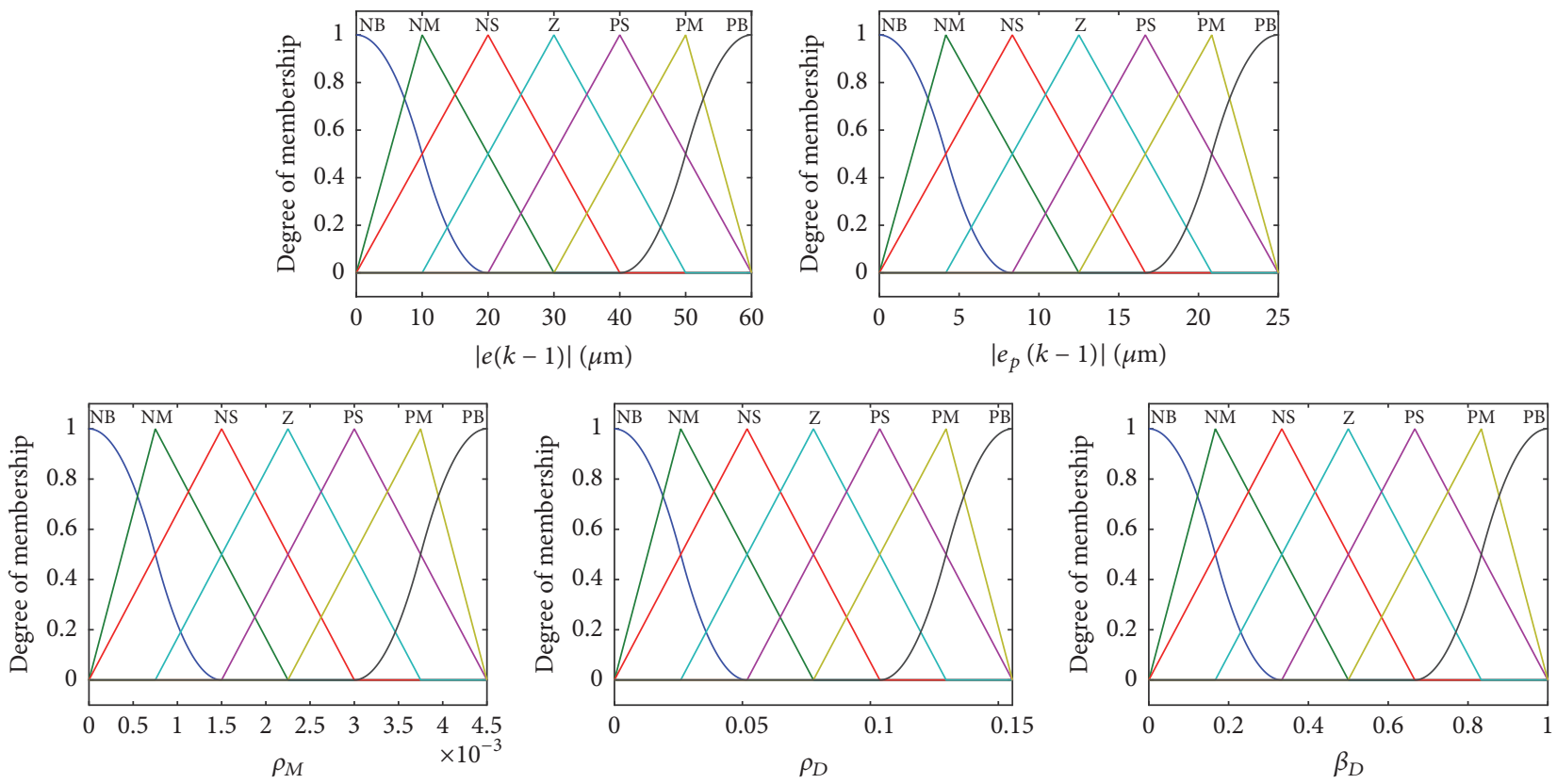

FIgURE 6: The membership functions of FDDMFAC when $y_{0}=60$.

TABLE 5: The fuzzy rules of $\rho_{D}, \beta_{D}$.

\begin{tabular}{|c|c|c|c|c|c|c|c|}
\hline \multirow{2}{*}{$\begin{array}{l}\rho_{D}, \beta_{D} \\
|e(k-1)|\end{array}$} & \multicolumn{7}{|c|}{$\left|e_{p}(k-1)\right|$} \\
\hline & NB & NM & NS & Z & PS & PM & PB \\
\hline NB & NB & NB & NB & NB & NB & NB & NB \\
\hline NM & NB & NM & NM & NM & $\mathrm{NM}$ & NS & NS \\
\hline NS & NM & NM & NM & NS & NS & $\mathrm{Z}$ & Z \\
\hline Z & Z & $\mathrm{Z}$ & $\mathrm{Z}$ & PS & PM & PM & PM \\
\hline PS & PS & PS & $\mathrm{PM}$ & $\mathrm{PM}$ & PB & $\mathrm{PB}$ & $\mathrm{PB}$ \\
\hline PM & $\mathrm{PM}$ & $\mathrm{PM}$ & $\mathrm{PM}$ & $\mathrm{PB}$ & $\mathrm{PB}$ & $\mathrm{PB}$ & $\mathrm{PB}$ \\
\hline PB & PB & $\mathrm{PB}$ & $\mathrm{PB}$ & $\mathrm{PB}$ & $\mathrm{PB}$ & $\mathrm{PB}$ & PB \\
\hline
\end{tabular}

validates the effectiveness of FDDMFAC proposed in this paper.

Although FDDMFAC approach has been proven to have a better control performance than MFAC and DDOILC, we still have a question of whether it is possible that the superiority of FDDMFAC is entirely attributed to fuzzy adjustment of parameters and has nothing to do with the structure of DDMFAC itself. In order to clarify this problem, another set of simulations are conducted, in which the control performance of FDDMFAC is compared with fuzzy MFAC (FMFAC) and fuzzy DDOILC (FDDOILC). The absolute value of tracking error $|e(k-1)|$ is regarded as the input variable of fuzzy rules and the step-size factors $\rho_{1}, \rho_{2}$ of MFAC and DDOILC are the output variables. The fuzzy rules of FMFAC and FDDOILC are shown in Table 6, and the membership functions when $y_{0}=60$ are shown in Figure 8. For the simulation results, please see Figure 9. From the simulation, it is concluded that FDDMFAC achieves the best
TABLE 6: The fuzzy rules of FMFAC and FDDOILC.

\begin{tabular}{llllllll}
\hline$|e(k-1)|$ & NB & NM & NS & Z & PS & PM & PB \\
\hline$\rho_{1}, \rho_{2}$ & NB & NM & NS & Z & PS & PM & PB \\
\hline
\end{tabular}

control performance, which demonstrates the effectiveness of the proposed approach in this paper.

\section{Conclusions}

This paper aimed at solving the problem of particle quality control in the drug development phase of SFBGP. The model-based control approaches are incapable of handling such quality control because the accurate process model cannot be acquired. Thus, the data-driven and model-free control approaches are considered in this work. We firstly 

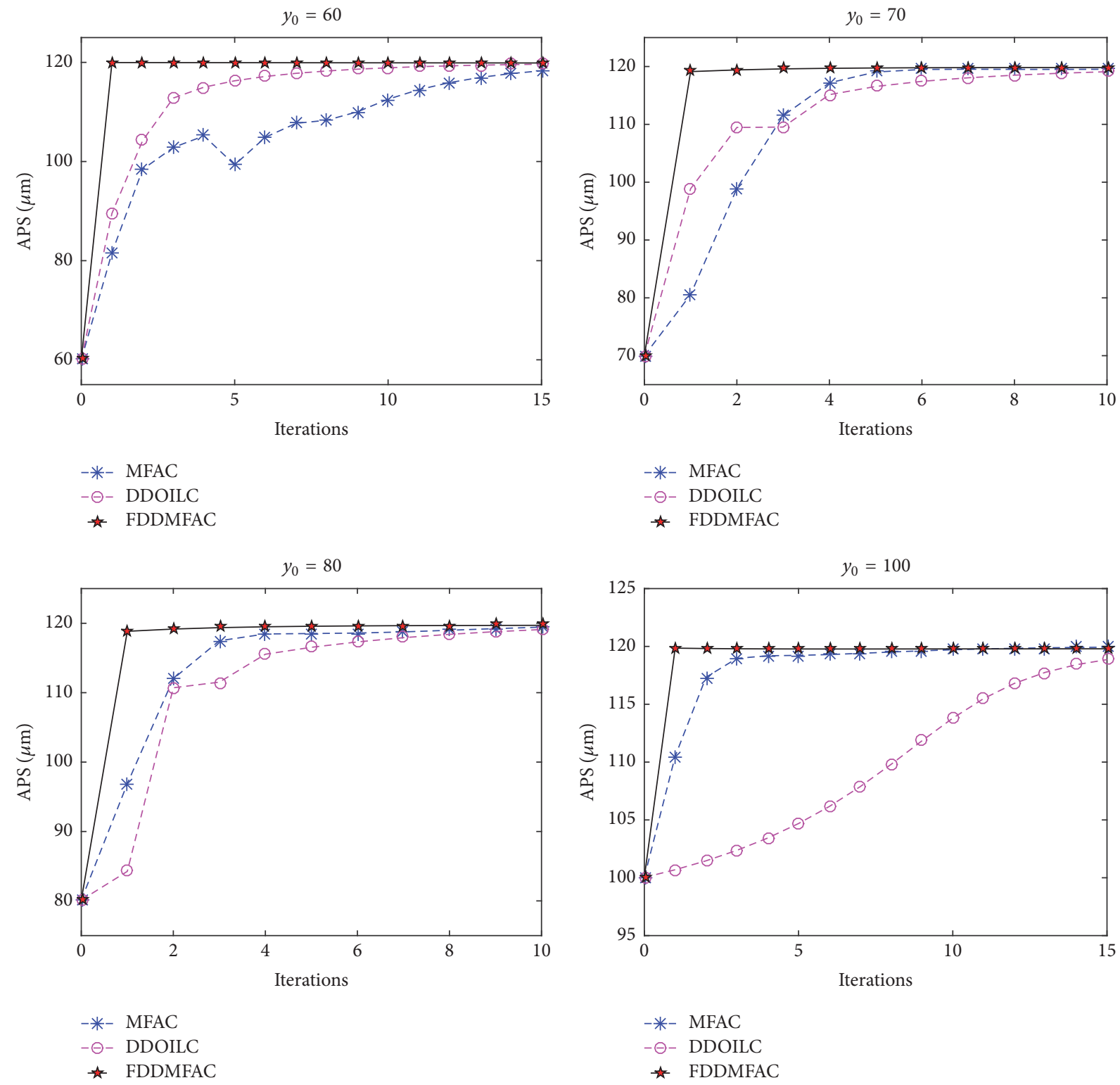

FIGURE 7: The comparison of control effects of MFAC, DDOILC, and FDDMFAC.

analyzed the features of MFAC and DDOILC and then conducted experimental research to compare the performances of MFAC and DDOILC in particle quality control under different IVs. The simulation results have verified our theoretical analysis and concluded that MFAC and DDOILC both have advantages under different IVs. To be used in an actual production environment, the expected DDC approaches must accommodate various IVs because of the changes in material attributes. Thus, the hybrid approach, FDDMFAC, which combines the advantages of MFAC and DDOILC by adaptively adjusting their respective weighted factors with fuzzy logic, was proposed in this work to complement their respective shortcomings. Through a series of simulation results, we conclude that FDDMFAC achieves better control performance than other approaches, which validates the effectiveness of FDDMFAC.

\section{Conflicts of Interest}

The authors declare that they have no conflicts of interest.

\section{Acknowledgments}

Thanks are due to National Natural Science Foundation of China under Grant nos. 61773105, 61374147, 61703085, and 61004083 and Fundamental Research Funds for the Central 

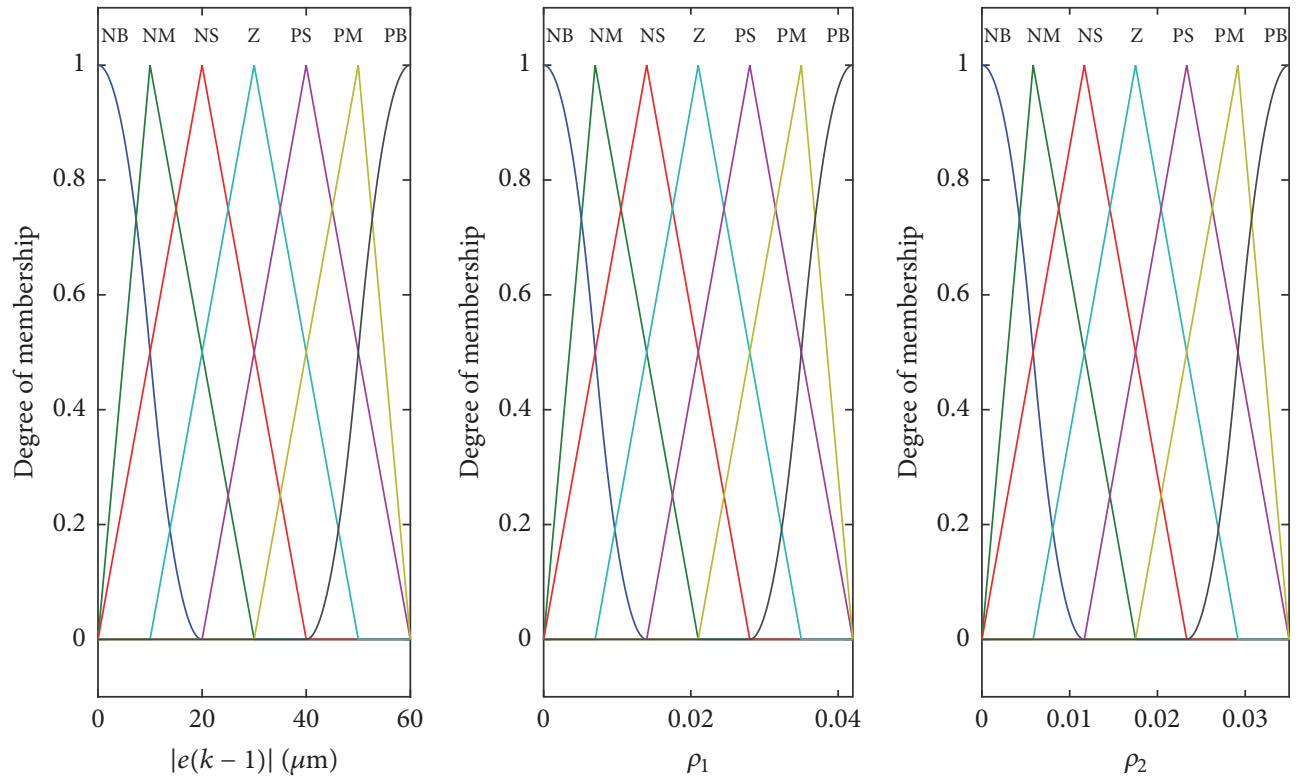

FIGURE 8: The membership functions of FDDMFAC when $y_{0}=60$.
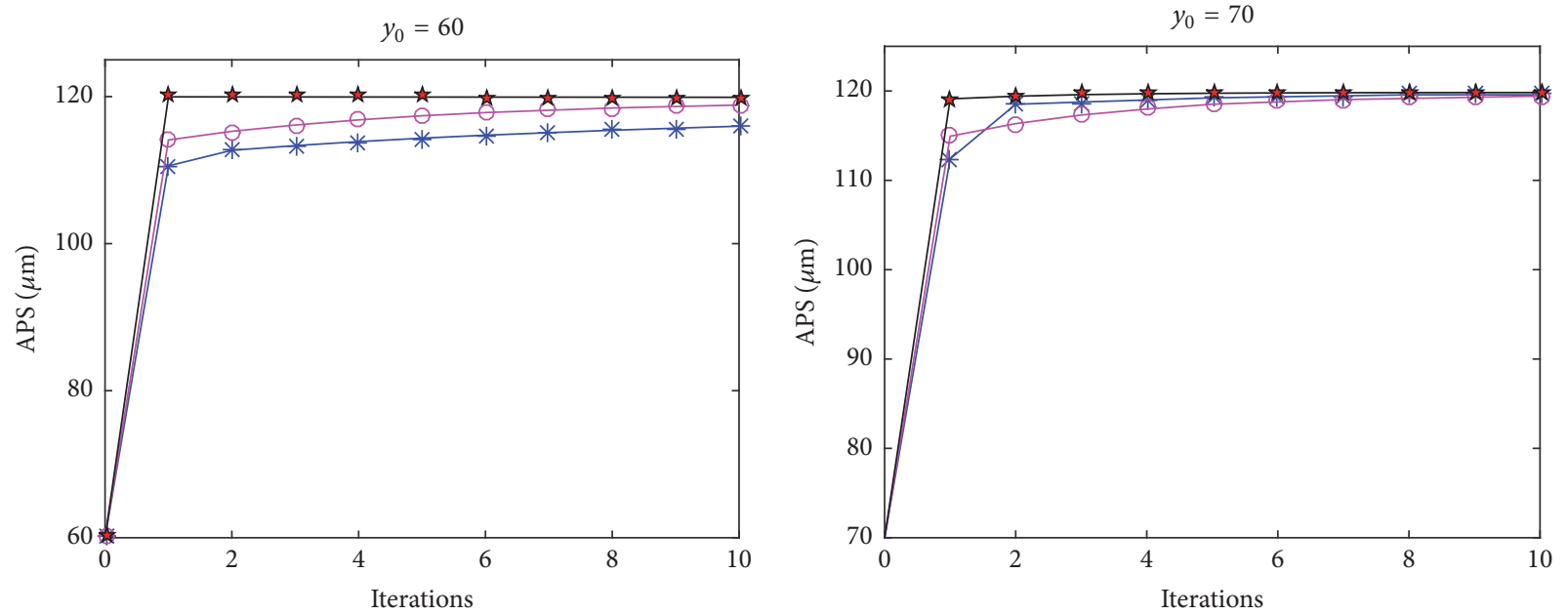

* FMFAC

๑ FDDOILC

* FDDMFAC
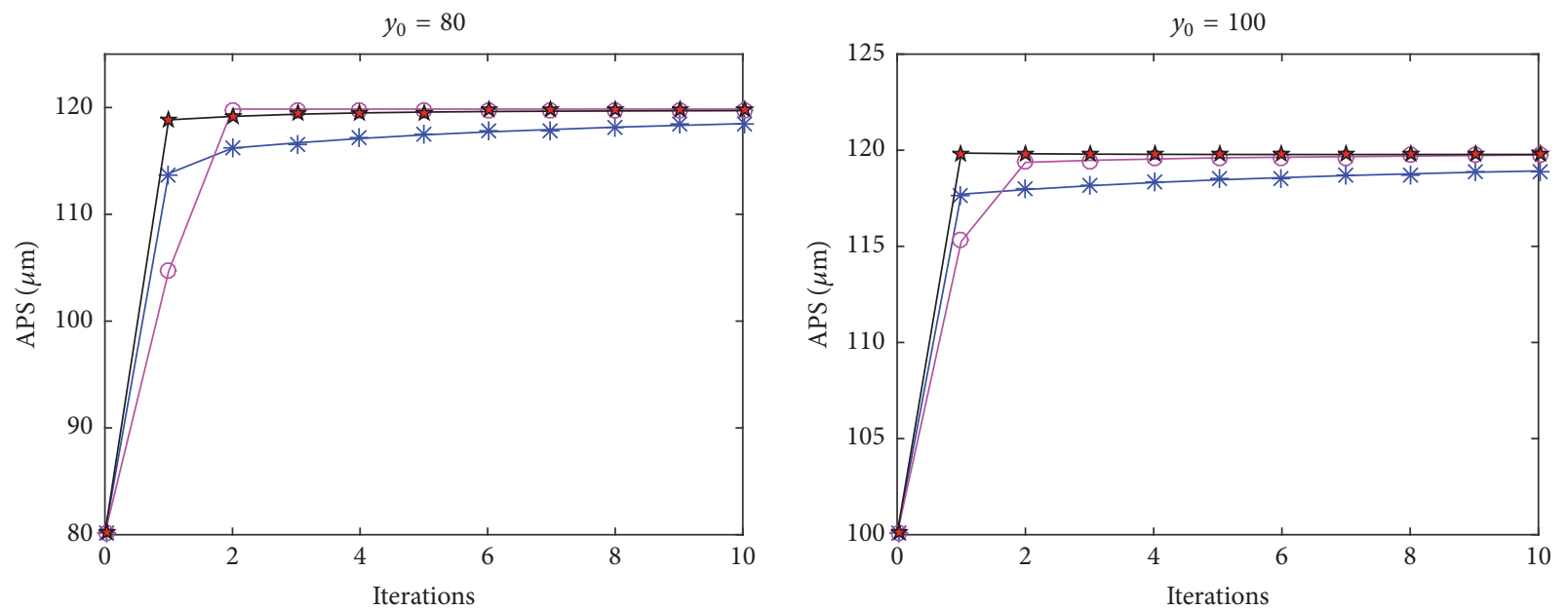

* FMFAC

$\because$ FDDOILC

* FDDMFAC

* FMFAC

$\odot$ FDDOILC

* FDDMFAC

FIGURE 9: The comparison of control effects of FMFAC, FDDOILC, and FDDMFAC. 
Universities under Grant nos. N150402001 and N120404014, for supporting this research work.

\section{References}

[1] J. Bouffard, M. Kaster, and H. Dumont, "Influence of process variable and physicochemical properties on the granulation mechanism of mannitol in a fluid bed top spray granulator," Drug Development and Industrial Pharmacy, vol. 31, no. 9, pp. 923-933, 2005.

[2] H. Liu, K. Wang, W. Schlindwein, and M. Li, "Using the BoxBehnken experimental design to optimise operating parameters in pulsed spray fluidised bed granulation," International Journal of Pharmaceutics, vol. 448, no. 2, pp. 329-338, 2013.

[3] H. Liu and M. Li, "Population balance modelling and multistage optimal control of a pulsed spray fluidized bed granulation," International Journal of Pharmaceutics, vol. 468, no. 1-2, pp. 223-233, 2014.

[4] T. Albuquerque, V. H. Dias, N. Poellinger, and J. F. Pinto, "Construction of a quality index for granules produced by fluidized bed technology and application of the correspondence analysis as a discriminant procedure," European Journal of Pharmaceutics and Biopharmaceutics, vol. 75, no. 3, pp. 418-424, 2010.

[5] H. Ehlers, A. Liu, H. Räikkönen et al., "Granule size control and targeting in pulsed spray fluid bed granulation," International Journal of Pharmaceutics, vol. 377, no. 1-2, pp. 9-15, 2009.

[6] C. F. W. Sanders, M. J. Hounslow, and F. J. Doyle III, "Identification of models for control of wet granulation," Powder Technology, vol. 188, no. 3, pp. 255-263, 2009.

[7] T. Närvänen, T. Lipsanen, O. Antikainen, H. Räikkönen, and J. Yliruusi, "Controlling granule size by granulation liquid feed pulsing," International Journal of Pharmaceutics, vol. 357, no. 1-2, pp. 132-138, 2008.

[8] A. Faure, P. York, and R. C. Rowe, "Process control and scaleup of pharmaceutical wet granulation processes: a review," European Journal of Pharmaceutics and Biopharmaceutics, vol. 52, no. 3, pp. 269-277, 2001.

[9] M. Pottmann, B. A. Ogunnaike, A. A. Adetayo, and B. J. Ennis, "Model-based control of a granulation system," Powder Technology, vol. 108, no. 2-3, pp. 192-201, 2000.

[10] E. P. Gatzke and F. J. Doyle III, "Model predictive control of a granulation system using soft output constraints and prioritized control objectives," Powder Technology, vol. 121, no. 2-3, pp. 149$158,2001$.

[11] F. Y. Wang and I. T. Cameron, "Review and future directions in the modelling and control of continuous drum granulation," Powder Technology, vol. 124, no. 3, pp. 238-253, 2002.

[12] T. Glaser, C. F. W. Sanders, F. Y. Wang et al., "Model predictive control of continuous drum granulation," Journal of Process Control, vol. 19, no. 4, pp. 615-622, 2009.

[13] R. Ramachandran and A. Chaudhury, "Model-based design and control of a continuous drum granulation process," Chemical Engineering Research and Design, vol. 90, no. 8, pp. 1063-1073, 2012.

[14] Z. H. Loh, D. Z. L. Er, L. W. Chan, C. V. Liew, and P. W. S. Heng, "Spray granulation for drug formulation," Expert Opinion on Drug Delivery, vol. 8, no. 12, pp. 1645-1661, 2011.

[15] S. H. Schaafsma, N. W. F. Kossen, M. T. Mos, L. Blauw, and A. C. Hoffmann, "Effects and control of humidity and particle mixing in fluid-bed granulation," AIChE Journal, vol. 45, no. 6 , pp. 1202-1210, 1999.
[16] Z.-S. Hou and S.-T. Jin, "A novel data-driven control approach for a class of discrete-time nonlinear systems," IEEE Transactions on Control Systems Technology, vol. 19, no. 6, pp. 1549-1558, 2011.

[17] Z. Hou and S. Jin, "Data-driven model-free adaptive control for a class of MIMO nonlinear discrete-time systems," IEEE Transactions on Neural Networks and Learning Systems, vol. 22, no. 12, pp. 2173-2188, 2011.

[18] D. Xu, B. Jiang, and P. Shi, "A novel model-free adaptive control design for multivariable industrial processes," IEEE Transactions on Industrial Electronics, vol. 61, no. 11, pp. 63916398, 2014.

[19] X. Wang, X. Li, J. Wang, X. Fang, and X. Zhu, "Data-driven model-free adaptive sliding mode control for the multi degreeof-freedom robotic exoskeleton," Information Sciences, vol. 327, pp. 246-257, 2016.

[20] L. dos Santos Coelho and A. A. R. Coelho, "Model-free adaptive control optimization using a chaotic particle swarm approach," Chaos, Solitons \& Fractals, vol. 41, no. 4, pp. 2001-2009, 2009.

[21] L. D. S. Coelho, M. W. Pessôa, R. Rodrigues Sumar, and A. Augusto Rodrigues Coelho, "Model-free adaptive control design using evolutionary-neural compensator," Expert Systems with Applications, vol. 37, no. 1, pp. 499-508, 2010.

[22] K. K. Tan, T. H. Lee, S. N. Huang, and F. M. Leu, "Adaptivepredictive control of a class of SISO nonlinear systems," Dynamics and Control, vol. 11, no. 2, pp. 151-174, 2001.

[23] B. Zhang and W. Zhang, "Adaptive predictive functional control of a class of nonlinear systems," ISA Transactions ${ }^{\circledR}$, vol. 45, no. 2, pp. 175-183, 2006.

[24] F. L. Lü, J. F. Wang, C. J. Fan, and S. B. Chen, "An improved model-free adaptive control with $G$ function fuzzy reasoning regulation design and its applications," Proceedings of the Institution of Mechanical Engineers, Part I: Journal of Systems and Control Engineering, vol. 222, no. 8, pp. 817-828, 2008.

[25] R. Chi, D. Wang, Z. Hou, and S. Jin, "Data-driven optimal terminal iterative learning control," Journal of Process Control, vol. 22, no. 10, pp. 2026-2037, 2012.

[26] R. Chi, Z. Hou, S. Jin, and D. Wang, "Improved data-driven optimal TILC using time-varying input signals," Journal of Process Control, vol. 24, no. 12, pp. 78-85, 2014.

[27] X. Bu, Z. Hou, and R. Chi, "Model free adaptive iterative learning control for farm vehicle path tracking," IFAC Proceedings Volumes, vol. 46, no. 20, pp. 153-158, 2013.

[28] M. C. Campi, A. Lecchini, and S. M. Savaresi, "Virtual reference feedback tuning: a direct method for the design of feedback controllers," Automatica, vol. 38, no. 8, pp. 1337-1346, 2002.

[29] R.-C. Roman, M.-B. Radac, R.-E. Precup, and E. M. Petriu, "Data-driven model-free adaptive control tuned by virtual reference feedback tuning," Acta Polytechnica Hungarica, vol. 13, no. 1, pp. 83-96, 2016.

[30] P. Yan, D. Liu, D. Wang, and H. Ma, "Data-driven controller design for general MIMO nonlinear systems via virtual reference feedback tuning and neural networks," Neurocomputing, vol. 171, pp. 815-825, 2016.

[31] T. Pan and S. Li, "A hierarchical search and updating database strategy for lazy learning," International Journal of Innovative Computing, Information and Control, vol. 4, no. 6, pp. 1383-1392, 2008.

[32] H. Zhang, R. Song, Q. Wei, and T. Zhang, "Optimal tracking control for a class of nonlinear discrete-time systems with time delays based on heuristic dynamic programming," IEEE 
Transactions on Neural Networks and Learning Systems, vol. 22, no. 12, pp. 1851-1862, 2011.

[33] J. D. Rojas and R. Vilanova, "Data-driven based IMC control," International Journal of Innovative Computing, Information and Control, vol. 8, no. 3, pp. 1557-1574, 2012.

[34] C. Li, J. Yi, and T. Wang, "Encoding prior knowledge into data driven design of interval type-2 fuzzy logic systems," International Journal of Innovative Computing Information and Control, vol. 7, no. 3, pp. 1133-1144, 2012.

[35] M.-B. Radac and R.-E. Precup, "Optimal behaviour prediction using a primitive-based data-driven model-free iterative learning control approach," Computers in Industry, vol. 74, pp. 95109, 2015.

[36] M.-B. Radac, R.-E. Precup, and E. . Petriu, "Model-free primitive-based iterative learning control approach to trajectory tracking of MIMO systems with experimental validation," IEEE Transactions on Neural Networks and Learning Systems, vol. 26, no. 11, pp. 2925-2938, 2015.

[37] M. Hussain, J. Kumar, M. Peglow, and E. Tsotsas, "Modeling spray fluidized bed aggregation kinetics on the basis of MonteCarlo simulation results," Chemical Engineering Science, vol. 101, pp. 35-45, 2013.

[38] M. Hussain, M. Peglow, E. Tsotsas, and J. Kumar, "Modeling of aggregation kernel using Monte Carlo simulations of spray fluidized bed agglomeration," AIChE Journal, vol. 60, no. 3, pp. 855-868, 2014.

[39] H. Liu and M. Li, "Two-compartmental population balance modeling of a pulsed spray fluidized bed granulation based on computational fluid dynamics (CFD) analysis," International Journal of Pharmaceutics, vol. 475, no. 1, pp. e256-e269, 2014.

[40] G. K. Reynolds, J. S. Fu, Y. S. Cheong, M. J. Hounslow, and A. D. Salman, "Breakage in granulation: a review," Chemical Engineering Science, vol. 60, no. 14, pp. 3969-3992, 2005.

[41] M. Peglow, J. Kumar, G. Warnecke, S. Heinrich, and L. Mörl, "A new technique to determine rate constants for growth and agglomeration with size- and time-dependent nuclei formation," Chemical Engineering Science, vol. 61, no. 1, pp. 282-292, 2006.

[42] M. J. Hounslow, R. L. Ryall, and V. R. Marshall, "A discretized population balance for nucleation, growth, and aggregation," AIChE Journal, vol. 34, no. 11, pp. 1821-1832, 1988.

[43] M. J. Hounslow, J. M. K. Pearson, and T. Instone, "Tracer studies of high-shear granulation: II. Population balance modeling," AIChE Journal, vol. 47, no. 9, pp. 1984-1999, 2001.

[44] G. C. Goodwin and K. S. Sin, Adaptive Filtering Prediction and Control, vol. 33, Prentice-Hall, Upper Saddle River, NJ, USA, 1984.

[45] D. He, Z. Wang, L. Yang, and Z. Mao, "Optimization control of the color-coating production process for model uncertainty," Computational Intelligence and Neuroscience, vol. 2016, Article ID 9731823, 12 pages, 2016. 


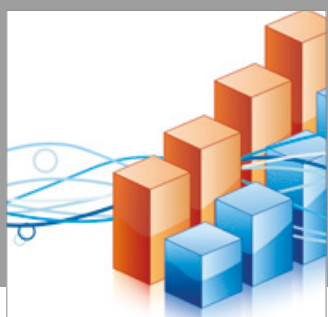

Advances in

Operations Research

vatersals

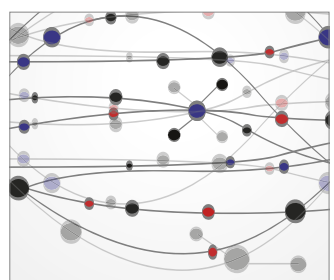

\section{The Scientific} World Journal
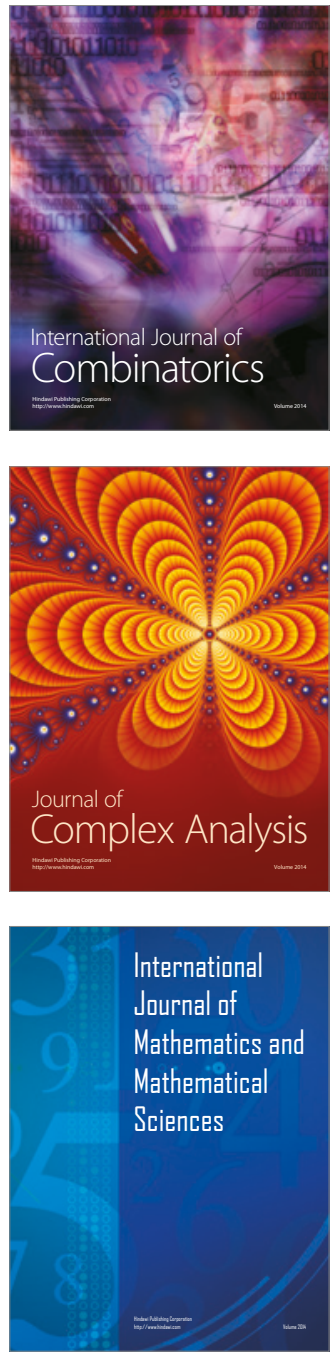
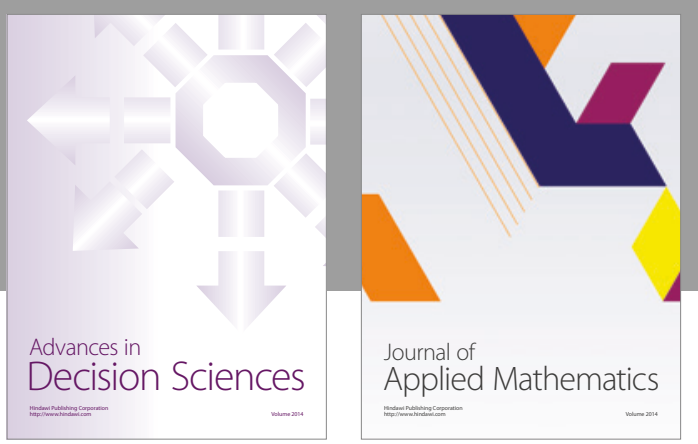

Algebra

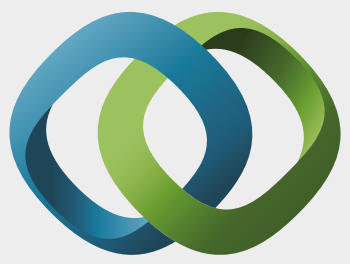

\section{Hindawi}

Submit your manuscripts at

https://www.hindawi.com
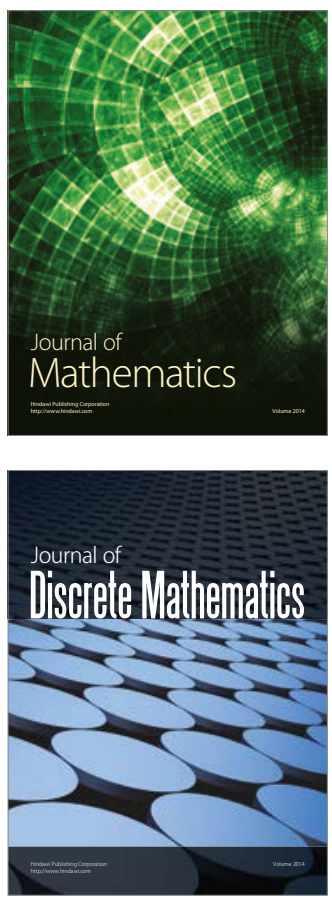

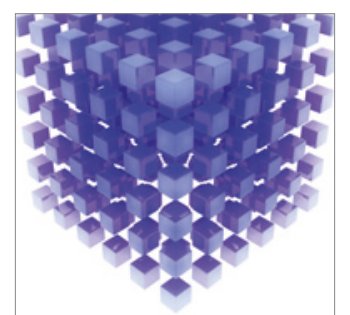

Mathematical Problems in Engineering
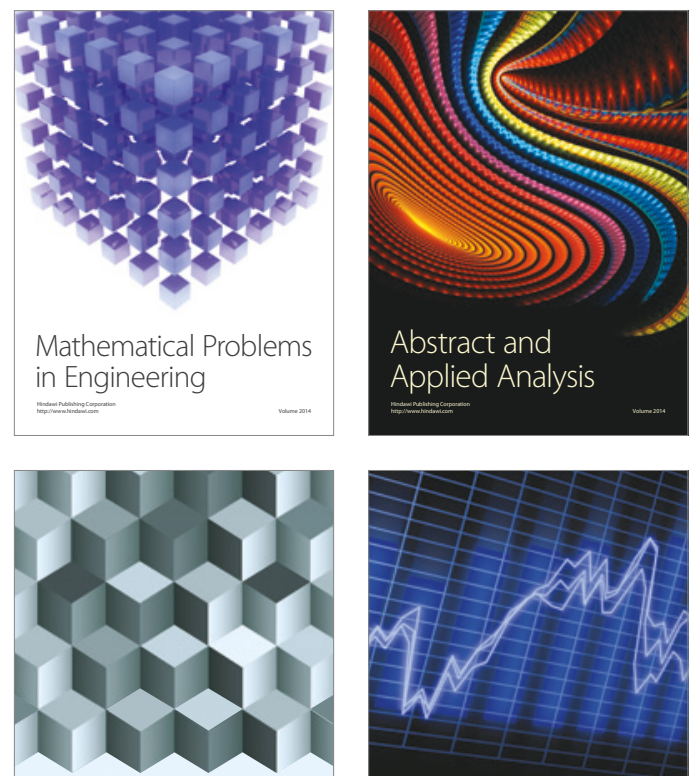

Journal of

Function Spaces

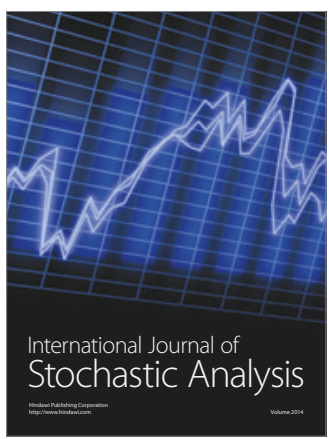

Probability and Statistics
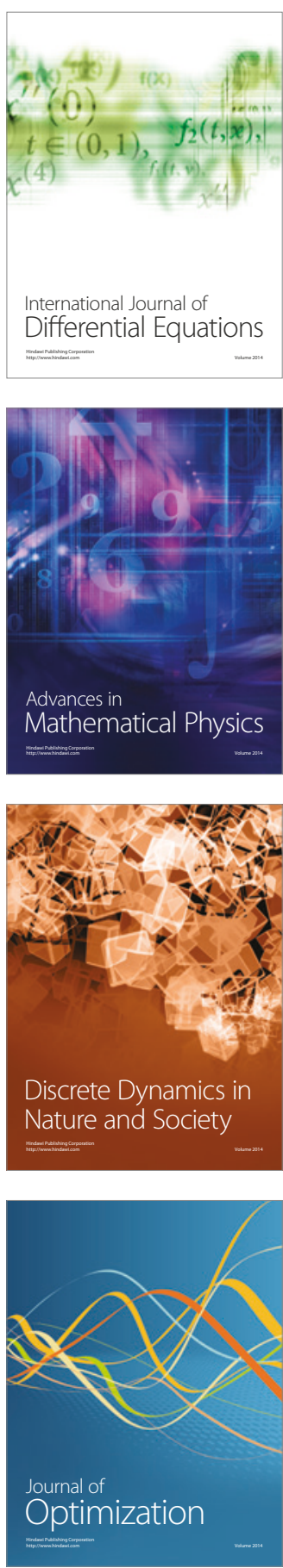